\title{
Spatial structure and coherent motion in dense planetary rings induced by self-gravitational instability
}

\author{
Hiroshi Daisaka and Shigeru Ida \\ Department of Earth and Planetary Sciences, Faculty of Science, Tokyo Institute of Technology, Tokyo 152-8551, Japan
}

(Received December 1, 1998; Revised April 6, 1999; Accepted May 10, 1999)

\begin{abstract}
We investigate the formation of spatial structure in dense, self-gravitating particle systems such as Saturn's B-ring through local $N$-body simulations to clarify the intrinsic physics based on individual particle motion. In such a system, Salo (1995) showed that the formation of spatial structure such as wake-like structure and particle grouping (clump) arises spontaneously due to gravitational instability and the radial velocity dispersion increases as the formation of the wake structure. However, intrinsic physics of the phenomena has not been clarified. We performed local $N$-body simulations including mutual gravitational forces between ring particles as well as direct (inelastic) collisions with identical (up to $N \sim 40000$ ) particles. In the wake structure particles no longer move randomly but coherently. We found that particle motion was similar to Keplerian motion even in the wake structure and that the coherent motion was produced since the particles in a clump had similar eccentricity and longitude of perihelion. This coherent motion causes the increase and oscillation in the radial velocity dispersion. The mean velocity dispersion is rather larger in a more dissipative case with a smaller restitution coefficient and/or a larger surface density since the coherence is stronger in the more dissipative case. Our simulations showed that the wavelength of the wake structure was approximately given by the longest wavelength $\lambda_{\text {cr }}=4 \pi^{2} G \Sigma / \kappa^{2}$ in the linear theory of axisymmetric gravitational instability in a thin disk, where $G, \Sigma$, and $\kappa$ are the gravitational constant, surface density, and a epicyclic frequency.
\end{abstract}

\section{Introduction}

The observation by the Voyager revealed that Saturn's ring is not homogeneous but it has complex structures. The ring has fine axisymmetric subrings with the width $\sim 10 \mathrm{~km}$ (e.g., Smith et al., 1982). Furthermore, the observation of Photopolarimeter (PPS) stellar occultation showed the finer density structure (microstructure) with scale down to $100 \mathrm{~m}$ in B-ring (Esposito et al., 1983a,b). Several ideas have been proposed to explain why such structure is formed and maintained against viscous diffusion due to collisions and gravitational scatterings of ring particles, but there is no satisfactory explanation.

To account for the axisymmetric subring structure, viscous instability was proposed by Lin and Bodenheimer (1981), Ward (1981), and Lukkari (1981). If viscous instability arises, diffusion from a less dense region is larger than that from a denser region so that the density contrast is strengthen until non-linear effect becomes important. The condition of this instability is $\partial(v \Sigma) / \partial \Sigma<0$, where $v$ and $\Sigma$ are kinematic viscosity and surface density of ring. It has been investigated whether a ring particle system satisfies the condition or not. However, negative results for viscous instability were reported by both theoretical studies using Boltzmann equation (Araki and Tremaine, 1986; Araki, 1988, 1991) and local $N$-body simulations (Wisdom and Tremaine, 1988; Salo, 1991, 1992a; Richardson, 1994).

Copy right (C) The Society of Geomagnetism and Earth, Planetary and Space Sciences (SGEPSS); The Seismological Society of Japan; The Volcanological Society of Japan; The Geodetic Society of Japan; The Japanese Society for Planetary Sciences.
On the other hand, Salo (1992b), Richardson (1994), and Salo (1995) gave important suggestions on the microstructure observed in B-ring. They extended the local $N$-body simulations developed by Wisdom and Tremaine (1988) to include self-gravitational forces between particles as well as inelastic collisions and used larger number of particles $(N>1000)$ than that in Wisdom and Tremaine (1988) $(N=40)$. They found the wake-like structure which may correspond to the substructure. Salo (1995) showed that the wakes are created if both self-gravity and inelastic collisions are included. Only self-gravity or only inelastic collision does not create such structure. The wake structure is time dependent and transient, being created and destroyed on the time scale of an order of Keplerian period. The wake structure looks like fluid turbulence.

Salo (1995) also found that when the wake-like structure is created, the equilibrium velocity dispersion of ring particles always satisfies $\mathcal{Q} \sim 2$, where $\mathcal{Q}$ is Toomre's nondimensional parameter (Toomre, 1964) defined by

$$
\mathcal{Q}=\frac{\kappa c_{r}}{3.36 G \Sigma}
$$

where $G$ is the gravitational constant, $\kappa$ is a epicyclic frequency, and $c_{r}$ is radial velocity dispersion.

Since the wake structure does not develop when $\mathcal{Q}>2$, self-gravitational instability would be responsible for the wake structure. The linear calculation shows that a selfgravitating, differentially rotating disk of collisionless particles becomes unstable against axisymmetric perturbation for $\mathcal{Q}<1$ (e.g., Toomre, 1964; Julian and Toomre, 1966). 
For non-axisymmetric perturbation, Griv (1998) derived a similar criterion, $\mathcal{Q} \sim 2 \Omega_{0} / \kappa$, where $\Omega_{0}$ is the angular velocity. Griv (1998) also confirmed the above criterion by local $N$-body simulations.

Since the relation of $\mathcal{Q} \sim 2$ is maintained once the wakelike structure is formed (Salo, 1995), the radial velocity dispersion $c_{r}$ increases with $\Sigma$. This increase in the radial velocity dispersion must be closely related with the formation of the wake structure. Salo (1995) suggested that such an increase of the velocity dispersion would come from scatterings by the collective wakes. He also suggested that the systematic motion of particles in the wakes may be responsible for the increase in the radial velocity dispersion. However, it has not been clarified how the radial velocity increase is related with the formation of the wake structure.

The study on the wake structure is important to clarify the microstructure in Saturn's B-ring. Furthermore, it is also important in the issue of the stability of Saturn's B-ring, since the transient wake structure would induce relatively large angular momentum flux to quickly diffuse out the ring. (The detailed analysis of the angular momentum transfer will be done in the next paper.)

We perform local $N$-body simulations including both mutual gravitational forces between particles and inelastic collisions as Salo (1995) did. We focus on the problem of the relation between the wake formation and the velocity increase through detailed analysis about how self-gravitating particles behave in the wake-like structure.

Simulation method is described in Section 2. In Section 3, we first compare our results with previous works (especially with Salo, 1995), then we will analyze the coherent particle motion in more detail. We find regular oscillation of the velocity dispersion associated with the increase in the time averaged velocity dispersion and clarify the relationship between the wake structure and the velocity change. We also analyze the scale of the structure by performing simulations with up to $N \sim 40000$ particles. In Section 4 we summarize the results and give discussion.

\section{Numerical Methods}

\subsection{Model description}

We adopt local $N$-body method which was first applied for the study of a dense ring system by Wisdom and Tremaine (1988) and followed by Salo (1991, 1992a,b, 1995) and Richardson (1994). The "local" means that we consider a box with width $L_{x}$ and height $L_{y}$ at a semimajor axis $a_{0}$ in the ring, which revolves in a circular orbit with the Kepler angular velocity $\Omega_{0}$ at the reference point $a_{0}$ and is small compared to the width of the whole ring (see Fig. 1). Motion of particles is pursued only in this box with the periodic boundary conditions. This method would be valid because we are considering the structure with much smaller scale than the width of the B-ring and the orbits of ring particles are nearly circular in the coplanar plane.

We use the Cartesian coordinates at $a_{0}$ which rotate with the angular velocity $\Omega_{0}$, in which $x$-axis points radially outward, $y$-axis points to the direction of orbital motion of the coordinate origin, and $z$-axis points to the direction normal to the orbital plane. Motion of a ring particle $i$ is described by

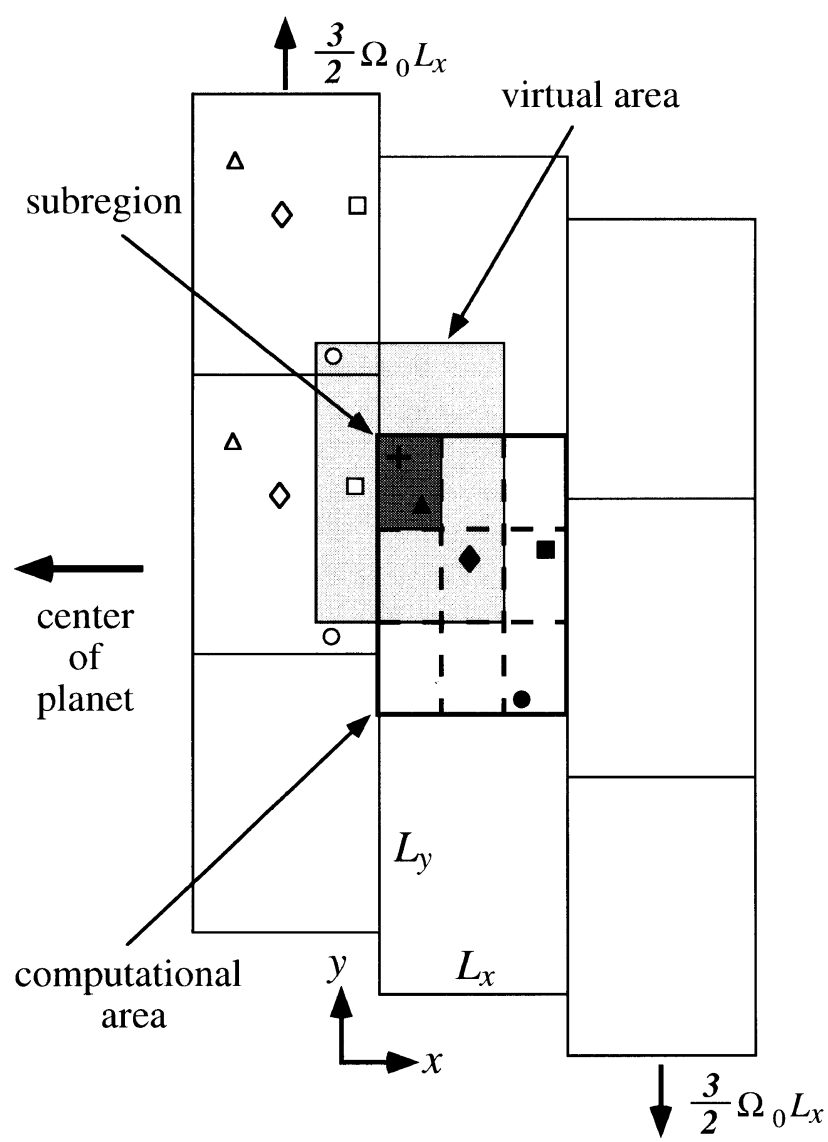

Fig. 1. Schematic illustration of computational domain. Original domain is a box with thick line. It is surrounded by eight copied domains. The shear velocities of the outside and inside boxes are $-3 \Omega_{0} L_{x} / 2$ and $+3 \Omega_{0} L_{x} / 2$. Computational area is divided into nine subareas (broken lines) and we make a virtual area for each subregion in which the subarea is centered. We calculate gravitational forces in the virtual area. For example, consider the particle denoted by a cross in the subregion (represented by dark shaded region). This subregion has virtual region represented by light shaded region.

Hill's equation (e.g., Hill, 1878; Nakazawa and Ida, 1988):

$$
\begin{aligned}
& \ddot{x}_{i}=2 \Omega_{0} \dot{y}_{i}+3 \Omega_{0}^{2} x_{i}+\sum_{j \neq i}^{N} \frac{G m_{j}}{r_{i j}^{3}}\left(x_{j}-x_{i}\right), \\
& \ddot{y}_{i}=-2 \Omega_{0} \dot{x}_{i}+\sum_{j \neq i}^{N} \frac{G m_{j}}{r_{i j}^{3}}\left(y_{j}-y_{i}\right), \\
& \ddot{z}_{i}=\quad-\Omega_{0}^{2} z_{i}+\sum_{j \neq i}^{N} \frac{G m_{j}}{r_{i j}^{3}}\left(z_{j}-z_{i}\right),
\end{aligned}
$$

where $m_{j}$ and $r_{i j}$ are mass of particle $j$ and the relative distance between particle $i$ and $j$, and $\Omega_{0}=\sqrt{G M_{\mathrm{s}} / a_{0}^{3}}, M_{\mathrm{s}}$ is mass of a central planet. The first and second terms on the right hand sides of Eqs. (2) denote the Coriolis and the tidal force, and the last terms are the mutual gravitational force between ring particles. When the mutual gravitational force can be neglected, Eqs. (2) are solved analytically and the solution represents an orbit of the Keplerian motion (see Nakazawa and Ida, 1988).

Scaling and parameters which characterize a ring system are as follow. Throughout the present paper, we assume that 
all particles have the same radius $r_{\mathrm{p}}$ and mass $m$. The mutual Hill's (tidal) radius is defined by $r_{\mathrm{h}}=h a_{0}$, where $h$ is the reduced Hill's radius defined as

$$
h=\left(\frac{2 m}{3 M_{\mathrm{s}}}\right)^{1 / 3},
$$

$r_{h}$ represents the radius of potential wall between two particles at which central force (tidal force) and mutual gravitational force between the particles are balanced. Within the radius, mutual gravity dominates central force and vise verse. It is possible to rewrite Eqs. (2) as mass-independent forms by scaling length, time, and mass as

$$
\begin{aligned}
\tilde{x} & =x / r_{\mathrm{h}}, \\
\tilde{t} & =t \Omega_{0}, \\
\tilde{m} & =m / M_{\mathrm{s}} h^{3}=\frac{3}{2} .
\end{aligned}
$$

The scaled mass $\tilde{m}$ is independent of mass of particle. In our simulations, we use these scaled variables and solve nondimensional equations of Eqs. (2).

A ring system is characterized by two non-dimensional parameters, optical depth $\tau$ and the ratio $r_{\mathrm{h}} / 2 r_{\mathrm{p}}$. The dynamical optical depth is defined as

$$
\tau=\frac{N \pi r_{\mathrm{p}}^{2}}{L_{x} L_{y}}
$$

where $L_{x} \times L_{y}$ and $N$ are the domain area and total number of particles in the domain. The optical depth can be expressed as $\tau \sim t_{\mathrm{K}} / t_{\mathrm{c}}$ (e.g., Goldreich and Tremaine, 1982), where $t_{\mathrm{K}}$ and $t_{\mathrm{c}}$ are the Keplerian time and the mean collision time. If $\tau$ is large enough (typically, $\tau \gtrsim 1$ ), clumps tend to be formed due to collision damping and self-gravity, while the clump formation is inhibited by differential rotation of the Keplerian motion if $\tau<1$, as shown later.

The other parameter $r_{\mathrm{h}} / 2 r_{\mathrm{p}}$ is

$$
\frac{r_{\mathrm{h}}}{2 r_{\mathrm{p}}} \simeq 0.82\left(\frac{\rho}{900 \mathrm{~kg} / \mathrm{m}^{3}}\right)^{1 / 3}\left(\frac{a_{0}}{10^{8} \mathrm{~m}}\right),
$$

where Saturn's mass, $M_{\mathrm{s}}=5.69 \times 10^{26} \mathrm{~kg}$, is assumed and $\rho$ represents material density of a ring particle. Since $\pi\left(2 r_{p}\right)^{2}$ and $\pi r_{h}^{2}$ express geometrical and characteristic gravitational cross sections for small random velocity, this parameter regulates which mechanism dominates velocity changes, gravitational scatterings or direct collisions. If $r_{\mathrm{h}} / 2 r_{\mathrm{p}} \gtrsim 1$, gravitational scatterings is more effective than direct collisions and vice versa (Ohtsuki, 1999). For the B-ring, $r_{\mathrm{h}} / 2 r_{\mathrm{p}} \sim 1$, so that both gravitational scatterings and direct collisions are important. On the other hand, ratio $r_{\mathrm{h}} / 2 r_{\mathrm{p}}$ is also related to the efficiency of the self-gravity against the tidal force of the central planet. Ohtsuki (1993) showed that the tidal force inhibits formation of persisting aggregates bounded by selfgravity if $r_{\mathrm{h}} / 2 r_{\mathrm{p}}<1$. From Eq. (6), the condition $r_{\mathrm{h}} / 2 r_{\mathrm{p}}>1$ holds outside A-ring. The systems with the same $r_{\mathrm{h}} / 2 r_{\mathrm{p}}$ have the same dynamical condition, even if $a_{0}$ and $\rho$ are different. We examine the dependence of the wake and clump formations on $r_{\mathrm{h}} / 2 r_{\mathrm{p}}$ rather than on $a_{0}$ and $\rho$.

In our simulations, we set up initial conditions as follows. The positions of particles are randomly distributed in the simulation region, avoiding overlapping of particles. The velocities except for the shear velocity of individual particles $3 \Omega_{0} x_{i} / 2$ are chosen randomly in the limited range from 0 to $5 r_{\mathrm{h}} \Omega_{0}$, but when the initial random velocity yields $\mathcal{Q}_{\text {initial }}<2$ we adopt random velocity large enough to be $\mathcal{Q}_{\text {initial }}>2$. The density structure obtained by our simulations is independent of choice of random numbers used to generate these initial conditions. The initial velocity is immediately relaxed and the equilibrium velocity state is established within a few Keplerian periods.

Equations (2) is integrated with the fourth-order Hermite scheme, which is one of predictor-corrector integrators and needs time derivatives of the mutual gravitational force as well as the mutual gravitational forces instead of past few data of position and velocity for interpolation (Makino and Aarseth, 1992). This integrator is easily implemented to the simulations in which there are discontinuous phenomenon such as direct collisions and jumps due to the periodic boundary conditions, because the Hermite integrator does not need data of past steps. In the integration, most expensive part of integration is the calculation of the mutual gravitational forces and its time derivatives whose calculation cost is $O\left(N^{2}\right)$. To reduce computational time, mutual force and its time derivative are calculated by HARP-2, which is a special purpose hardware for calculating gravitational force (Makino et al., 1993). "HARP” means Hermite AcceleratoR Pipeline and is one kind of GRAPE system ("GRAPE" means GRAvity PipE; Sugimoto et al., 1990). This hardware is connected to a host computer through a communication interface. The host sends position, velocity, and mass of all particles to the hardware. The hardware calculates gravitational forces with pipelines and returns them to the host. The host integrates orbits utilizing the returned gravitational forces. By using this hardware, calculation cost is significantly reduced.

\subsection{Calculation of mutual gravitational force and direct collision}

As stated above, the mutual gravitational force between ring particles and the direct (inelastic) collision play an important role in the evolution and the kinetic behavior of particle system, and the wake and clump formations. An equilibrium state is established by the balance between viscous energy gain (heating) through gravitational scatterings and direct collisions and energy dissipation (cooling) through inelastic collisions.

In the original Wisdom and Tremaine's method, the mutual gravity was ignored or was only treated as vertical force by enhancement of vertical frequency. Salo (1992b, 1995) and Richardson (1994) extended simulations exactly including mutual force. Richardson (1994) used the tree method while Salo (1995) carried out direct sum of forces exerted on a particle from neighbor particles within the distance $R_{\max }$. The cut-off length $R_{\max }$ is often substantially less than width of simulation region. In our simulations, we adopt direct sum of forces from all particles with some adjustment explained below.

Salo (1995) studied the influence of cut-off distance $R_{\max }$ and found an optimal value. But the larger $R_{\max }$ may be needed when the strong wake-like structure appears and coherent motion in the structure is important. We consider gravitational forces exerted on a particle from all other par- 

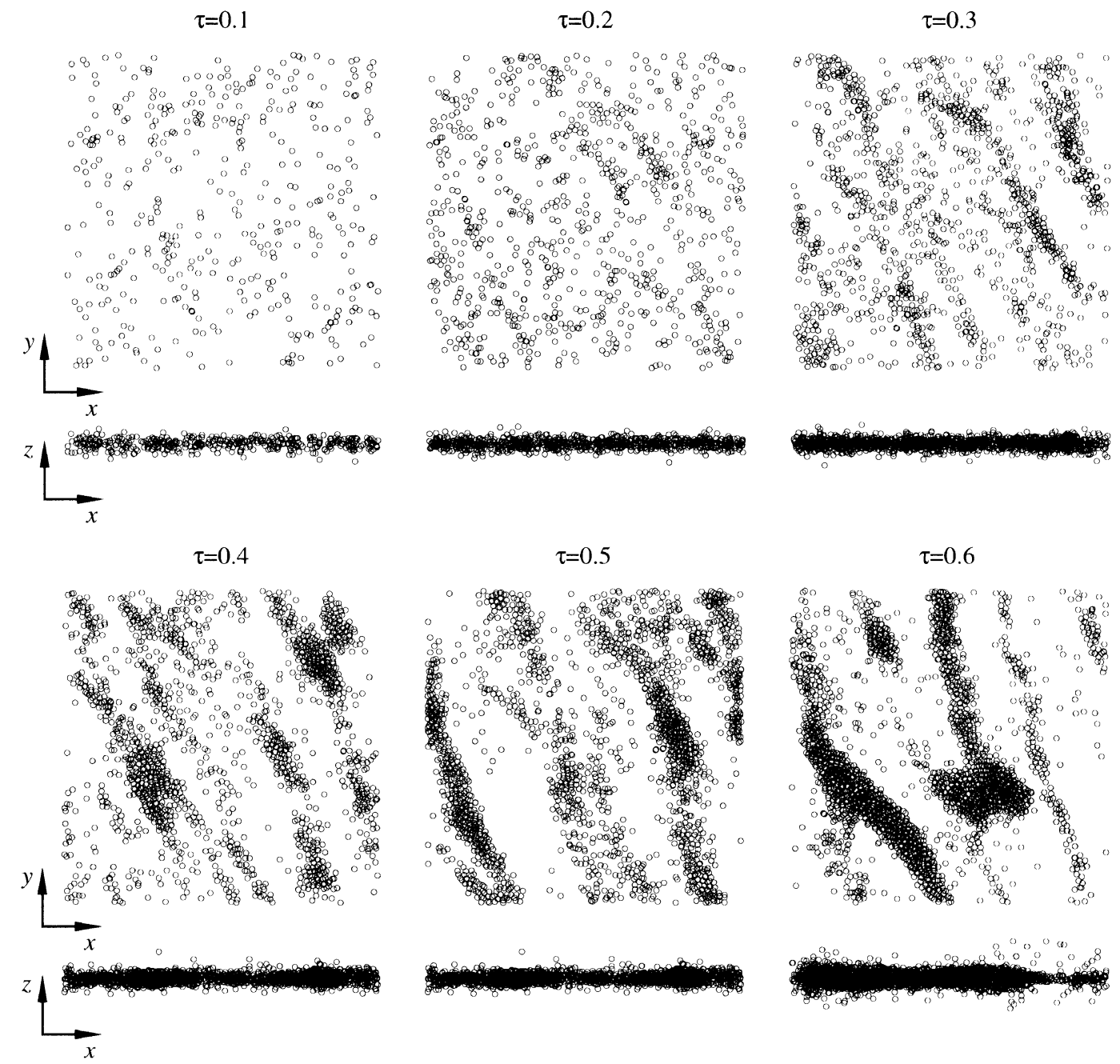

Fig. 2(a). Snapshots of the particle distribution with various optical depth ( $\tau=0.1$ to 0.6$)$. Upper and lower panels are face-on and edge-on distribution. In all cases, the restitution coefficient $\epsilon$ and ratio $r_{\mathrm{h}} / 2 r_{\mathrm{p}}$ are $\epsilon=0.5$ and $r_{\mathrm{h}} / 2 r_{\mathrm{p}}=0.82$, respectively. The width of the simulation region is $L=112 \mathrm{~m}$ $\left(L=68.3 r_{\mathrm{h}}\right)$.

ticles used in the simulation, carefully treating particles near boundary as well as in Salo (1995). For the effective use of HARP, we introduce the following subregion method. As in Fig. 1, we consider an original region (a box with thick line) and its copies. Because of the Keplerian shear velocity, radially inner and outer boxes have different angular velocity so that these boxes slide upward and downward with the shear velocity $3 \Omega_{0} L_{x} / 2$. A particle at $(x, y, z)$ in the central box with the velocity $\left(v_{x}, v_{y}, v_{z}\right)$ has its images at $\left(x \pm n L_{x}, y \pm m L_{y} \mp 3 \Omega_{0} n L_{x} t / 2, z\right)$ with the velocity $\left(v_{x}, v_{y} \mp 3 \Omega_{0} n L_{x} / 2, v_{z}\right)$, where $n$ and $m$ are integers. When a particle in the central box outgoes from a certain boundary, its image at the neighbor box comes from the opposite boundary. We divide the simulation region into nine subregions, represented as broken line in Fig. 1. For each subregion, we make virtual regions with the same size of original. In the virtual regions, the subregion is centered. Dark and light shaded regions in Fig. 1 are the example of a subregion and its virtual region. For particles in the subregion, we count gravitational forces of all particles in its virtual region. In this method, it happens that force from closer particle is neglected but that from more distant particle is counted. However, this asymmetry occurs in the outer part of the virtual region and does not affect the results because the size of the simulation is considerably larger than $R_{\max }$ Salo (1995) used. Actually we confirmed it performing the simulations with different size of simulation region.

In our simulations, we adopt a hard-sphere collision model that is commonly used in previous simulations (Wisdom and Tremaine, 1988; Salo, 1991, 1992a,b, 1995; Richardson, 1994). A collision changes only impact velocity in normal direction. Normal and tangential components of relative velocities of colliding particles after the collision, $v_{n}^{\prime}$ and $v_{t}^{\prime}$, are described by

$$
\begin{aligned}
& v_{n}^{\prime}=-\epsilon v_{n}, \\
& v_{t}^{\prime}=v_{t},
\end{aligned}
$$

where $\epsilon$ is the restitution coefficient of ring particles. Generally, $\epsilon$ depends on its material properties as well as the impact velocity. In spite of many efforts (Bridges et al., 1984; Hatzes et al., 1988; Dilley, 1993; Supulver et al., 1995), we have had no precise knowledge on $\epsilon$ yet. In this study, we will treat $\epsilon$ as a parameter.

Detection of collision is one of difficulties in treating col- 

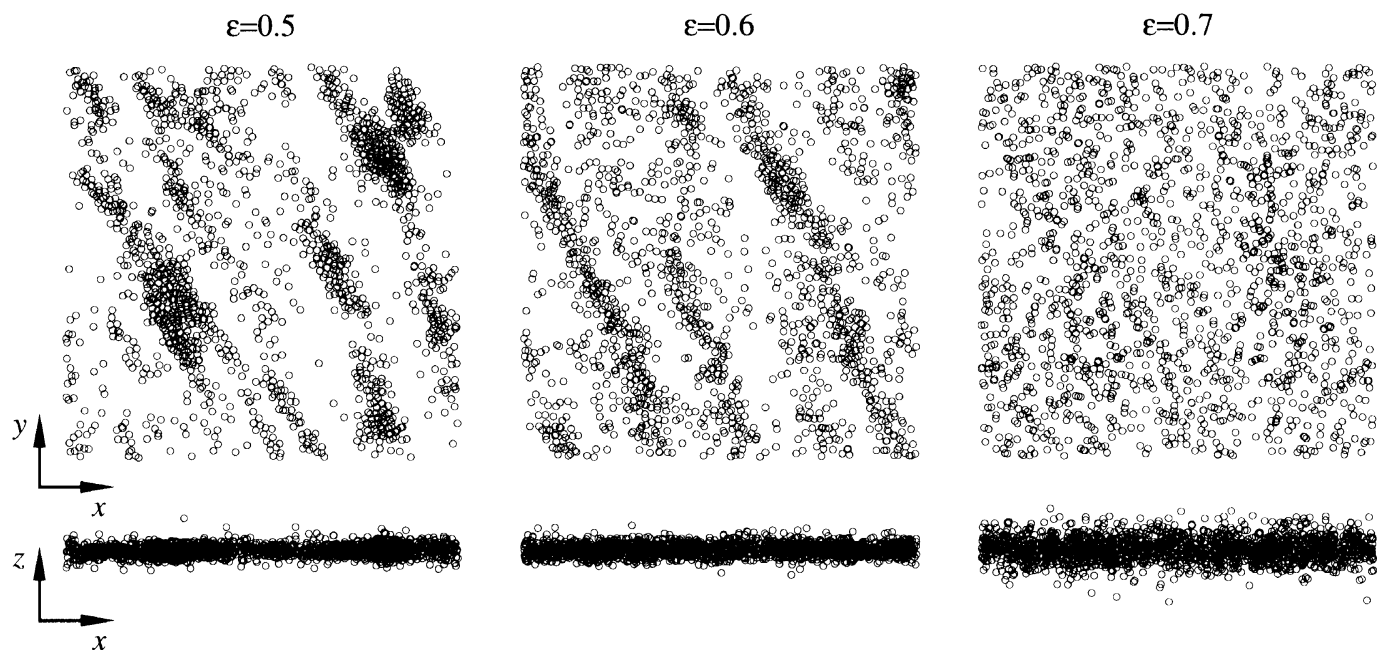

Fig. 2(b). The same as Fig. 2(a) but simulations with various restitution coefficient $(\epsilon=0.5,0.6$, and 0.7$)$ for $\tau=0.4$.
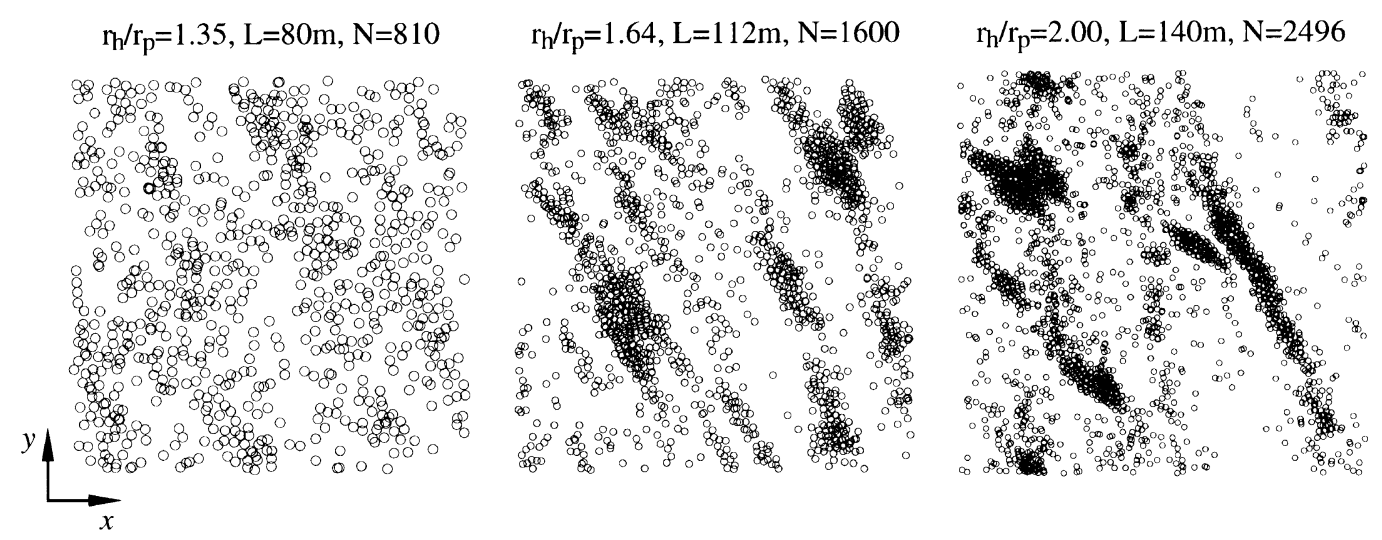

Fig. 2(c). The same as Figs. 2(a) and 2(b) but simulations with various $r_{\mathrm{h}} / 2 r_{\mathrm{p}}$ for $\tau=0.4$ and $\epsilon=0.5$. The width is set as $L=59.3 r_{\mathrm{h}}(L=80 \mathrm{~m})$ for $r_{\mathrm{h}} / 2 r_{\mathrm{p}}=0.675, L=68.3 r_{\mathrm{h}}(L=112 \mathrm{~m})$ for $r_{\mathrm{h}} / 2 r_{\mathrm{p}}=0.82$, and $L=70.0 r_{\mathrm{h}}(L=140 \mathrm{~m})$ for $r_{\mathrm{h}} / 2 r_{\mathrm{p}}=1.00$, respectively.

lisions. Ideally, collisions should be detected at the instance when colliding pairs just touch each other but it is practically impossible. Such problems were discussed by Richardson (1994) and Salo (1995). In this study, we use a simple method for detection of collision. Collision is detected as overlapping of colliding particles approaching each other. We search colliding pairs by using a neighbor list of each particle which is calculated by HARP-2 as well as mutual forces and its time derivatives. If a pair in the neighbor list overlaps, $r_{i j} \leq r_{\mathrm{p}, i}+r_{\mathrm{p}, j}$, and is approaching, $\boldsymbol{r}_{i j} \cdot \boldsymbol{v}_{i j}<0$, where $\boldsymbol{r}_{i j}$ and $\boldsymbol{v}_{i j}$ are relative distance $\boldsymbol{r}_{i j}=\boldsymbol{r}_{j}-\boldsymbol{r}_{i}$ and velocity $\boldsymbol{v}_{i j}=\boldsymbol{v}_{j}-\boldsymbol{v}_{i}$ and $r_{\mathrm{p}, i}$ is radius of particle $i$, we assume that these particles collide and velocity of colliding pair after the collision is calculated by Eqs. (7). If $r_{i j} \leq r_{\mathrm{p}, i}+r_{\mathrm{p}, j}$ but $\boldsymbol{r}_{i j} \cdot \boldsymbol{v}_{i j}>0$, we let the particles separate. However, colliding bodies can happen to overlap significantly without having been detected. In this case, the bodies keep sinking, which stops calculation (Richardson, 1994; Salo, 1995). To avoid this trouble, we remove overlapping of colliding bodies by the method used by Richardson (1994). In his method, overlapping bodies are moved outward along the line connecting each center to the location where these particles are just touching. From conservation of center of mass of colliding particles, adjusted positions are given as

$$
\begin{aligned}
& x_{i}^{\prime}=x_{i}-\Delta r \\
& x_{j}^{\prime}=x_{j}+\Delta r
\end{aligned}
$$

where the prime denotes position after correction and

$$
\Delta \boldsymbol{r}=\frac{r_{\mathrm{p}, i}+r_{\mathrm{p}, j}-r_{i j}}{2 r_{i j}} \boldsymbol{r}_{i j} .
$$

We always apply this adjustment when collision is detected.

The penetration depth of colliding bodies can be controlled by changing accuracy of timestep. In our simulations, we adopted shared, variable timestep because of frequent direct collisions and mutual gravitational forces. The formula of timestep is simply given by

$$
\delta t=\min _{i}\left(\alpha \frac{\left|\boldsymbol{a}_{i}\right|}{\left|\dot{\boldsymbol{a}}_{i}\right|}\right),
$$

where $\alpha$ is timestep coefficient and $\boldsymbol{a}_{i}$ and $\dot{\boldsymbol{a}}_{i}$ are acceleration of particle $i$ and its time derivative. 


\section{Results of Simulations}

\subsection{Comparison with Salo (1995)}

In this study, we restrict our simulations to identical particle systems in order to understand the basic dynamical properties. Previous simulations performed by Salo (1992b) and Richardson (1994) included the effect of a mass (size) distribution of ring particles so that our simulation results can not be compared directly with their results. On the other hand, Salo (1995) performed simulations of identical particle systems with a wider range of parameters and obtained statistical quantities such as radial velocity dispersion. First we perform simulations with the same parameters as used in Salo (1995) and confirm his results.

Salo (1995) found the spontaneous formation of the wake structure in identical particles if self-gravity of ring particles is effective as well as inelastic collision, while previous studies without the self-gravity by Wisdom and Tremaine (1988) and Salo (1991, 1992a) did not find such a structure. The structure is non-axisymmetric one such as collective JulianToomre wakes (Julian and Toomre, 1966). The formation of the wake structure should be related with gravitational instability. Salo (1995) performed simulations with various ring parameters such as $\tau, \epsilon, a_{0}$, and $\rho$ (last two correspond to $r_{\mathrm{h}} / 2 r_{\mathrm{p}}$ ) and showed that the formation of the wake structure is regulated by the condition of the Toomre parameter $\mathcal{Q}$ value for gravitational instability (Eq. (1)). Small $\epsilon$ and large $\tau$ lead to gravitational instability, because small $\epsilon$ results in small velocity dispersion and large $\tau$ results in large surface density. For small $r_{\mathrm{h}} / 2 r_{\mathrm{p}}$, formation of persistent aggregates by accretion are suppressed by the tidal force.

Formation of spatial structure influences the motion of individual particles. Salo (1995) showed that as the wake structure grows, the radial velocity dispersion increases with a large magnitude of its fluctuation and tends to keep the relation $\mathcal{Q} \simeq 2$. Salo (1995) also discussed the equilibrium radial velocity dispersion. He showed that when $r_{\mathrm{h}} / 2 r_{\mathrm{p}}$ is small, i.e., the self-gravity is insignificant, the velocity
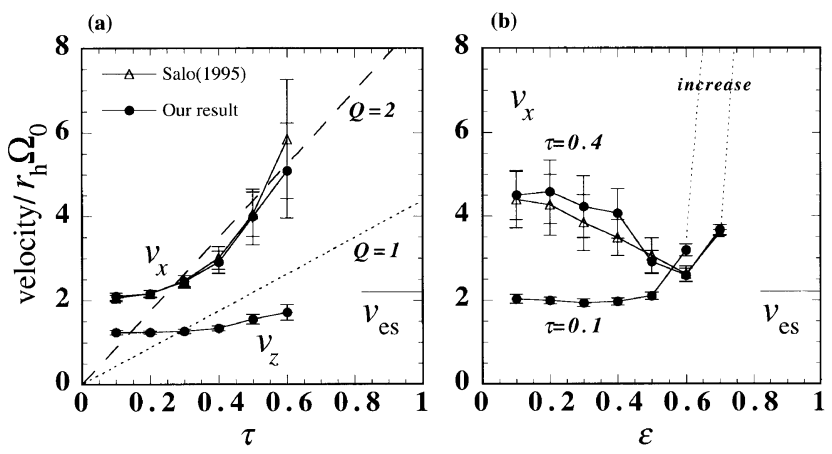

Fig. 3. Equilibrium values of the velocity dispersion as a function of the optical depth $\tau$ for $\epsilon=0.5$ (a) and the restitution coefficient $\epsilon$ for $\tau=0.4$ and $\tau=0.1$ (b). Our results and Salo(1995) are represented by filled circles and open triangles, respectively. For our results, both $\left\langle v_{x}^{2}\right\rangle^{1 / 2}$ and $\left\langle v_{z}^{2}\right\rangle^{1 / 2}$ are plotted in (a) but only $\left\langle v_{x}^{2}\right\rangle^{1 / 2}$ in (b). For Salo(1995), only $\left\langle v_{x}^{2}\right\rangle^{1 / 2}$ is plotted in both (a) and (b). Surface escape velocity is $v_{\mathrm{es}}=\sqrt{2 G m / r_{\mathrm{p}}}$, where $m$ and $r_{\mathrm{p}}$ are mass and radius of a particle, respectively. Both dotted and broken lines in (a) are calculated from Toomre's $\mathcal{Q}$-value (Eq. (1)) as being $\mathcal{Q}=1$ and $\mathcal{Q}=2$. All velocities are scaled by $r_{\mathrm{h}} \Omega_{0}$. dispersion is dominated by direct collisions rather than the self-gravity to have a value of an order $\sim r_{\mathrm{p}} \Omega$, but when $r_{\mathrm{h}} / 2 r_{\mathrm{p}}$ is large but the surface density is small enough to avoid gravitational instability, gravitational encounters dominate and the velocity dispersion attains the surface escape velocity $v_{\mathrm{es}}=\sqrt{2 G m / r_{\mathrm{p}}}$, where $m$ is mass of a particle. Thus, equilibrium velocity may be altered by the parameter which characterizes a ring system as well as inelasticity of a ring particle.

Our simulations show quite similar results to Salo (1995). Figure 2 is the spatial distribution of particles viewed face on and edge on. In Figs. 2(a) and 2(b), the optical depth $\tau$ is changed with fixed, velocity-independent coefficient restitution $\epsilon=0.5$ and $\epsilon$ is changed with fixed $\tau=0.4$, respectively. The other parameters are the same in all simulations; $r_{\mathrm{h}} / 2 r_{\mathrm{p}}=0.82$ corresponding to, for example, the situation with internal density $\rho_{0}=900 \mathrm{~kg} / \mathrm{m}^{3}$ and Saturncentric distance $a_{0}=1.0 \times 10^{8} \mathrm{~m}$, and $r_{\mathrm{p}}=1 \mathrm{~m}$. The simulation region is square with the width $L=112 \mathrm{~m}$ $\left(L=68.3 r_{\mathrm{h}}\right)$. The optical depth is controlled by changing number of particles, for examples, we use $N=400$ for $\tau=0.1$ and $N=2400$ for $\tau=0.6$, respectively. Figure $2(\mathrm{c})$ is the results of the simulations with various ratio $r_{\mathrm{h}} / 2 r_{\mathrm{p}}$ for $\tau=0.4$ and $\epsilon=0.5$. In these simulations, the region is also square and the width of the region and number

Table 1. Equilibrium radial velocity dispersions in simulations with parameters used by Salo (1995); simulations with various optical depth $\tau$ for $\epsilon=0.5, r_{\mathrm{h}} / 2 r_{\mathrm{p}}=0.82$.

\begin{tabular}{ccccc}
\hline & & & \multicolumn{2}{c}{$\left\langle v_{x}^{2}\right\rangle^{1 / 2} / r_{\mathrm{h}} \Omega_{0}$} \\
\cline { 4 - 5 }$\tau$ & $N$ & $L / \lambda_{\text {cr }}$ & Our results & Salo (1995) \\
\hline 0.1 & 400 & 13.5 & $2.094 \pm 0.084$ & $2.05 \pm 0.08$ \\
0.2 & 800 & 6.7 & $2.157 \pm 0.084$ & $2.16 \pm 0.08$ \\
0.3 & 1200 & 4.5 & $2.443 \pm 0.149$ & $2.46 \pm 0.13$ \\
0.4 & 1600 & 3.4 & $2.906 \pm 0.261$ & $3.01 \pm 0.27$ \\
0.5 & 2000 & 2.7 & $3.987 \pm 0.663$ & $4.05 \pm 0.53$ \\
0.6 & 2400 & 2.2 & $5.094 \pm 1.137$ & $5.84 \pm 1.42$ \\
\hline
\end{tabular}

Table 2. Simulations with various velocity-independent restitution coefficient $\epsilon$ for $\tau=0.4$ and $r_{\mathrm{h}} / 2 r_{\mathrm{p}}=0.82$.

\begin{tabular}{ccc}
\hline & \multicolumn{2}{c}{$\left\langle v_{x}^{2}\right\rangle^{1 / 2} / r_{\mathrm{h}} \Omega_{0}$} \\
\cline { 2 - 3 }$\epsilon$ & Our results & Salo (1995) \\
\hline 0.1 & $4.500 \pm 0.589$ & $4.39 \pm 0.671$ \\
0.2 & $4.578 \pm 0.762$ & $4.27 \pm 0.732$ \\
0.3 & $4.221 \pm 0.741$ & $3.84 \pm 0.671$ \\
0.4 & $4.059 \pm 0.589$ & $3.48 \pm 0.427$ \\
0.5 & $2.906 \pm 0.261$ & $3.05 \pm 0.427$ \\
0.6 & $2.584 \pm 0.168$ & $2.61 \pm 0.183$ \\
0.7 & $3.670 \pm 0.114$ & $3.60 \pm 0.073$ \\
\hline
\end{tabular}




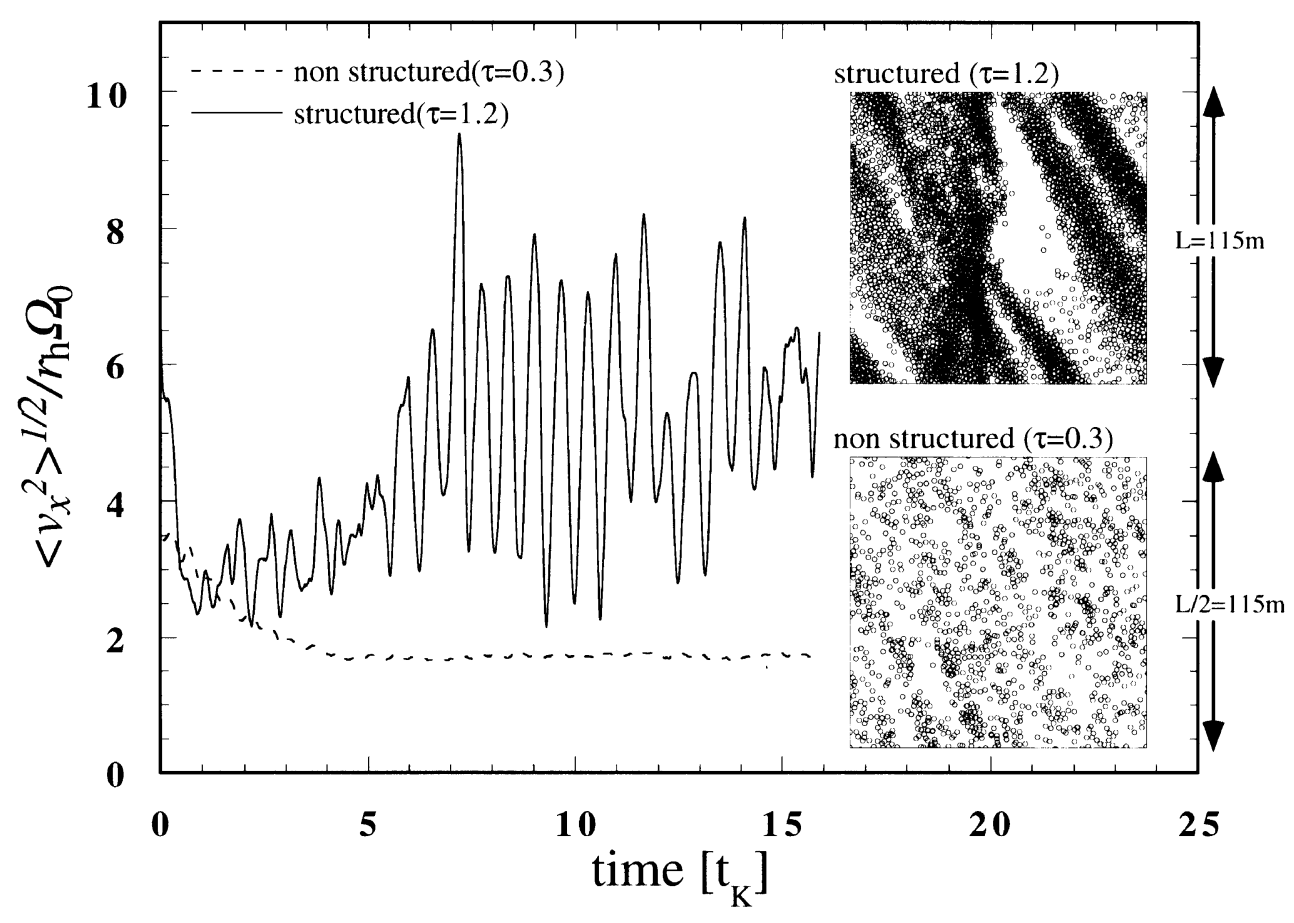

Fig. 4. Time evolution of the radial velocity dispersions for $\tau=0.3$ (broken line) and $\tau=1.2$ (solid line), which correspond to the non-structured and the structured cases, respectively. In both simulations, $\epsilon$ and $r_{\mathrm{h}} / 2 r_{\mathrm{p}}$ are $\epsilon=0.4$ and $r_{\mathrm{h}} / 2 r_{\mathrm{p}}=0.675$. The number of particles is $N=5052$ in both cases. Typical spatial distributions of both cases are superimposed. For $\tau=0.3$, edge of the distribution is trimmed away and the distribution displays the same area as that for $\tau=1.2$. The width of each box is $115 \mathrm{~m}\left(85.2 r_{\mathrm{h}}\right)$.

of particles are $L=59.3 r_{\mathrm{h}}(L=80 \mathrm{~m})$ and $N=810$ for $r_{\mathrm{h}} / 2 r_{\mathrm{p}}=0.675, L=68.3 r_{\mathrm{h}}(L=112 \mathrm{~m})$ and $N=1600$ for $r_{\mathrm{h}} / 2 r_{\mathrm{p}}=0.82$, and $L=70.0 r_{\mathrm{h}}(L=140 \mathrm{~m})$ and $N=2496$ for $r_{\mathrm{h}} / 2 r_{\mathrm{p}}=1.00$, respectively. These figures correspond to figures 10b, 11c, and 9c (and figure 14) in Salo (1995), respectively. Figure 2 shows that the increase of $\tau$ and/or ratio $r_{\mathrm{h}} / 2 r_{\mathrm{p}}$, and the decrease of $\epsilon$ lead to the formation of the strong wake structure, as pointed out by Salo (1995). This wake-like structure is formed after a few Keplerian periods from the beginning of the simulation. The wake structure changes with time. It is formed and dissolved successively on time scale of an order of a Keplerian period, so that the evolution of spatial structure affects the equilibrium velocity dispersion.

Figure 3 shows the equilibrium velocity of $\left\langle v_{x}^{2}\right\rangle^{1 / 2}$ and $\left\langle v_{z}^{2}\right\rangle^{1 / 2}$ as functions of $\tau$ and $\epsilon$ for the simulations of Figs. 2(a) and 2(b). In Fig. 3(b), we also show the results with $\tau=0.1$. Each equilibrium value and error bar are the time average and the fluctuation of each velocity component after initial relaxation (see Fig. 4). In simulations of Figs. 2 and 3, the initial relaxation time is a few Keplerian periods. As shown in Fig. 4, the dispersion $\left\langle v_{x}^{2}\right\rangle^{1 / 2}$ in the structured case $(\tau=1.2)$ oscillates regularly with time. In this case, the bars represent the amplitude of the oscillation. The dotted and dashed lines in Fig. 3(a) are the critical velocity calculated from Toomre's $\mathcal{Q}$ being $\mathcal{Q}=1$ and $\mathcal{Q}=2$, respectively. Tables 1 and 2 list the equilibrium values of $\left\langle v_{x}^{2}\right\rangle^{1 / 2}$ scaled by $r_{\mathrm{h}} \Omega_{0}$ obtained by Salo's and our simulations with various $\tau$ and $\epsilon$. In Fig. 3, filled circles and open triangles are our results and Salo's, respectively. They are in good agreement with each other.

From the comparison of Fig. 3 with Fig. 2, it is evident that the formation of the wake structure leads to the large increase in the equilibrium radial velocity dispersion with the large amplitude of its fluctuation, as pointed out by Salo (1995). On the other hand, any drastic change is not observed in vertical velocity dispersion $\left\langle v_{z}^{2}\right\rangle^{1 / 2}$ even if a strong wake structure is formed. Figure 3(a) shows that $\left\langle v_{x}^{2}\right\rangle^{1 / 2}$ increases with $\tau$, following the line of $\mathcal{Q}=2$. This tendency of $\left\langle v_{x}^{2}\right\rangle^{1 / 2}$ to follow $\mathcal{Q} \simeq 2$ is commonly observed in simulations with other parameters which allow the formation of the wake-like structure. Figure 3(b) shows that in the region $\epsilon \gtrsim 0.6$ for $\tau=0.4$ and $\epsilon \gtrsim 0.4$ for $\tau=0.1,\left\langle v_{x}^{2}\right\rangle^{1 / 2}$ is reduced as decreasing $\epsilon$ because the collisional dissipation becomes strong. Thickness of ring is also reduced as clearly seen in $x-z$ plot of particle distribution for $\epsilon=0.6$ and 0.7 in Fig. 2(b). On the contrary, in the region $\epsilon \lesssim 0.6$ for $\tau=0.4$, $\left\langle v_{x}^{2}\right\rangle^{1 / 2}$ increases with decrease in $\epsilon$. In the case of $\tau=0.1$, $\tau$ is too small to cause gravitational instability, so that the increase of $\left\langle v_{x}^{2}\right\rangle^{1 / 2}$ is not found. In this case, gravitational scatterings dominate and the equilibrium velocity is given by the surface escape velocity, as pointed out by Salo (1995). This tendency is also observed in Fig. 3(a) at small $\tau$. Note that in the case where $r_{\mathrm{h}} / 2 r_{\mathrm{p}}$ is small, the equilibrium velocity should be dominated by direct collision rather than by gravitational scattering. Similar results were obtained by Ohtsuki (1999), who investigated the equilibrium velocity dispersion in the dilute ring system $(\tau \ll 1)$ by numerical three-body integration of particle's orbits taking into account finite size of particles. When $\epsilon$ is sufficiently large $(\epsilon>0.7$ for $\tau=0.4, \epsilon>0.6$ for $\tau=0.1$ ), collisional damping cannot equilibrate with stirring due to gravitational scatterings and direct collisions, so that both $\left\langle v_{x}^{2}\right\rangle^{1 / 2}$ and $\left\langle v_{z}^{2}\right\rangle^{1 / 2}$ 
Table 3. Simulations with various number of particles for $\tau=1.2, \epsilon=0.4$, and $r_{\mathrm{h}} / 2 r_{\mathrm{p}}=0.675$.

\begin{tabular}{rcccccc}
\hline \multicolumn{1}{c}{$N$} & $L_{x} / r_{\mathrm{h}}$ & $L_{y} / r_{\mathrm{h}}$ & $L_{x} / \lambda_{\text {cr }}$ & $L_{y} / \lambda_{\text {cr }}$ & $v_{x} / r_{\mathrm{h}} \Omega_{0}$ & err \\
\hline 2460 & 60 & 60 & 1.45 & 1.45 & 5.45 & 1.61 \\
5054 & 85 & 85 & 2.07 & 2.07 & 5.28 & 1.49 \\
10696 & 60 & 260 & 1.45 & 6.27 & 6.12 & 1.51 \\
21290 & 120 & 260 & 2.90 & 6.27 & 7.16 & 1.38 \\
42780 & 240 & 260 & 5.80 & 6.27 & 6.55 & 0.712 \\
\hline
\end{tabular}

monotonically increase. These results approximately agrees with the critical restitution coefficient for the existence of a steady state obtained by Goldreich and Tremaine (1978) and Ohtsuki (1992, 1999).

Thus, our simulations as well as Salo (1995) show that the formation of the wake structure occurs in a dense ring system with self-gravitating particles and that as the spatial structure develops, the radial velocity dispersion increases with a large magnitude of fluctuation and takes $\mathcal{Q} \simeq 2$. Salo (1995) suggested that such increase of the velocity dispersion is due to scatterings by collective wakes. However, he did not give detailed argument. On the other hand, Salo (1995) suggested through the velocity field of particles in the wake that the velocity increase comes from the difference of systematic motions between adjacent wakes. Next we will analyze the motion of particles in detail to clarify the relation between the velocity increase and the wake structure.

3.2 Coherence of particle's motion in the wake structure

As shown in the preceding subsection, the formation and the evolution of the spatial structure and the motion of particles influence each other. To clarify the relation, we analyze motion of particles in the wake-like structure in detail.

First we compare the evolution of the radial velocity dispersion in the cases with and without wake-like structure. We perform two large $N$-body simulations for $\tau=0.3$ and $\tau=1.2$, which correspond to the non-structured and the structured cases, respectively (see spatial distributions in Fig. 4). The number of particles and the width of the square simulation region are $N=5052$ and $L=230 \mathrm{~m}$ ( $\left.L=170.5 r_{\mathrm{h}}, L / \lambda_{\mathrm{cr}}=16.54\right)$ for $\tau=0.3$, and $L=115 \mathrm{~m}$ ( $L=85.2 r_{\mathrm{h}}, L / \lambda_{\mathrm{cr}}=2.07$ ) for $\tau=1.2$. The other parameters are fixed as $\epsilon=0.4$ and $r_{\mathrm{h}} / 2 r_{\mathrm{p}}=0.675$. Figure 4 shows the evolution curves of the velocity dispersion $\left\langle v_{x}^{2}\right\rangle^{1 / 2}$ and the spatial distributions in two cases. For $\tau=0.3$, the wake structure is very weak because of low surface number density and $\left\langle v_{x}^{2}\right\rangle^{1 / 2}$ attains a steady state with a small magnitude of fluctuation after a few Keplerian periods. On the other hand, for $\tau=1.2$, the velocity dispersion increases with oscillation with a large amplitude after initial relaxation time $\left(\sim 1 t_{\mathrm{K}}\right)$. In the beginning of the simulation, $\left\langle v_{x}^{2}\right\rangle^{1 / 2}$ is rapidly reduced by the dissipation of random motion energy through inelastic collisions, and it becomes small $\sim 2.5 r_{\mathrm{h}} \Omega_{0}$. But, after $t=1 t_{\mathrm{K}},\left\langle v_{x}^{2}\right\rangle^{1 / 2}$ begins to increase, accompanied by oscillation. This velocity increase is closely associated with the formation of the strong wake structure.

Similar results are also observed in simulations with different sets of parameters in which wake structure is formed. To

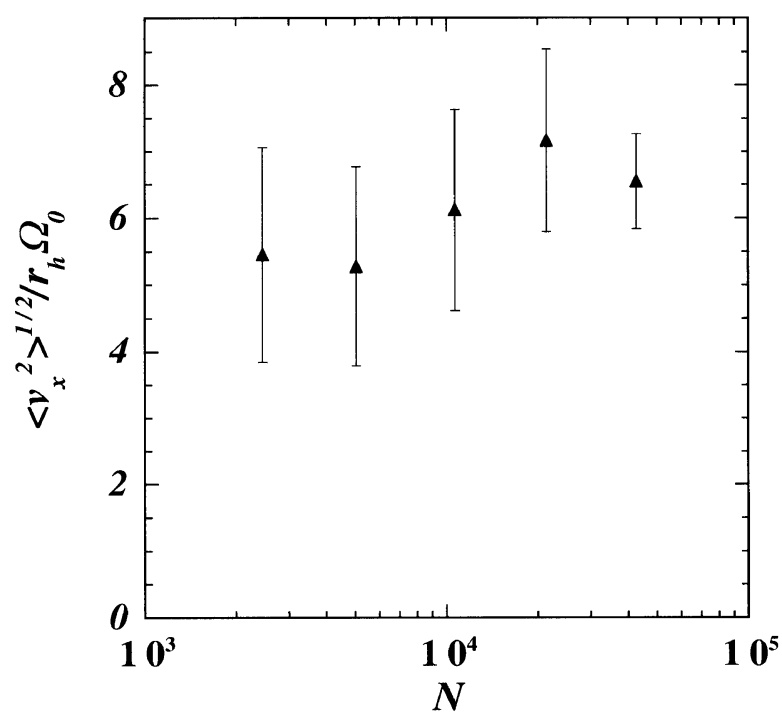

Fig. 5. Dependence of the velocity dispersion on number of particles (equivalently, area of the computational region). In each simulation, $\tau=1.2, \epsilon=0.4$, and $r_{\mathrm{h}} / 2 r_{\mathrm{p}}=0.675$.

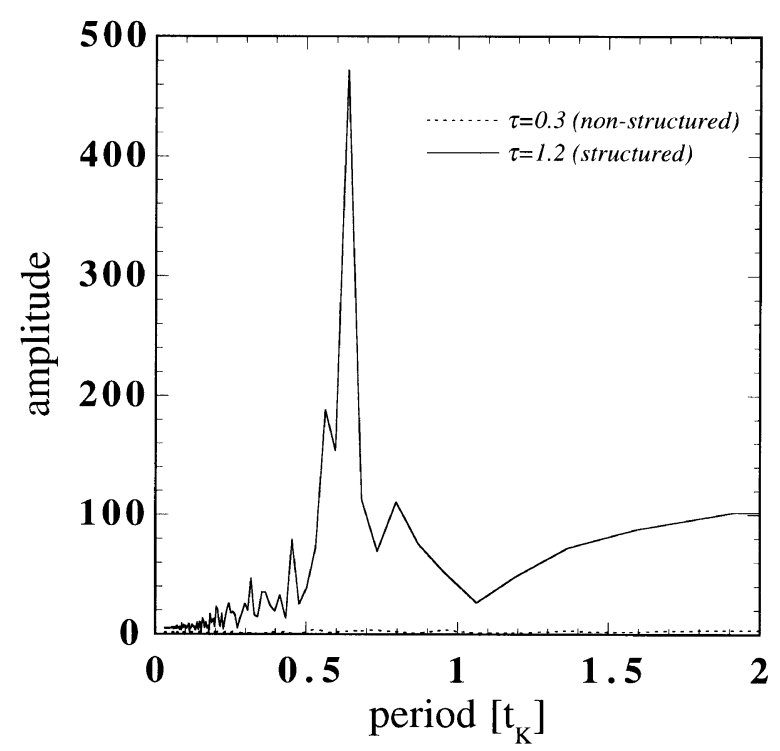

Fig. 6. Spectrum analysis of a period of oscillation observed in the evolution curve of $\left\langle v_{x}^{2}\right\rangle^{1 / 2}$ in the non-structured (broken line) and the structured cases (solid line). 
(a)

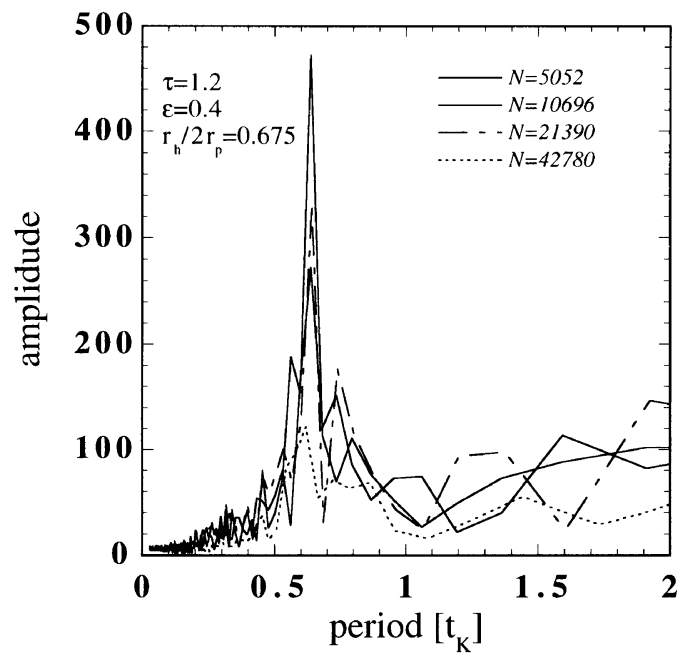

(c)

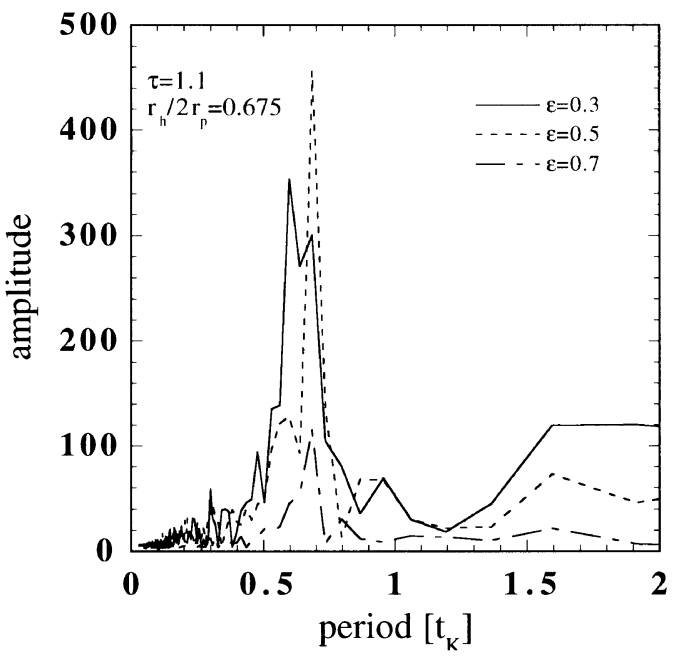

(b)

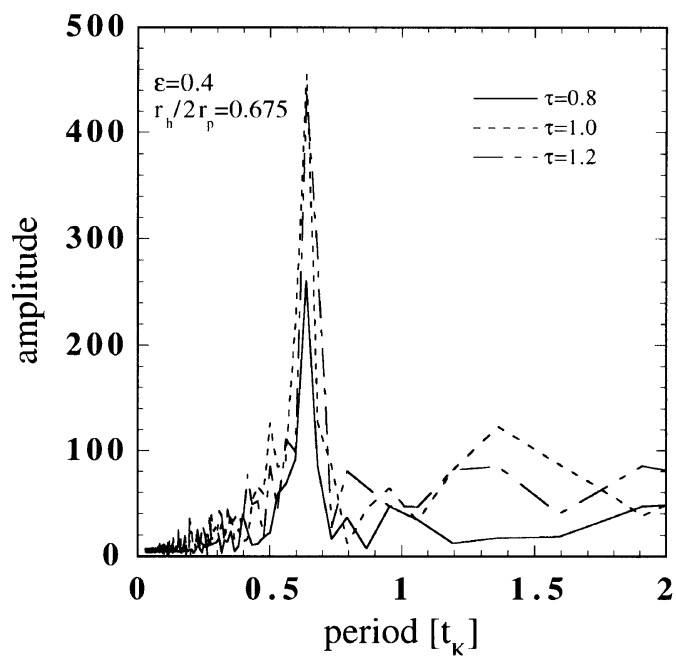

(d)

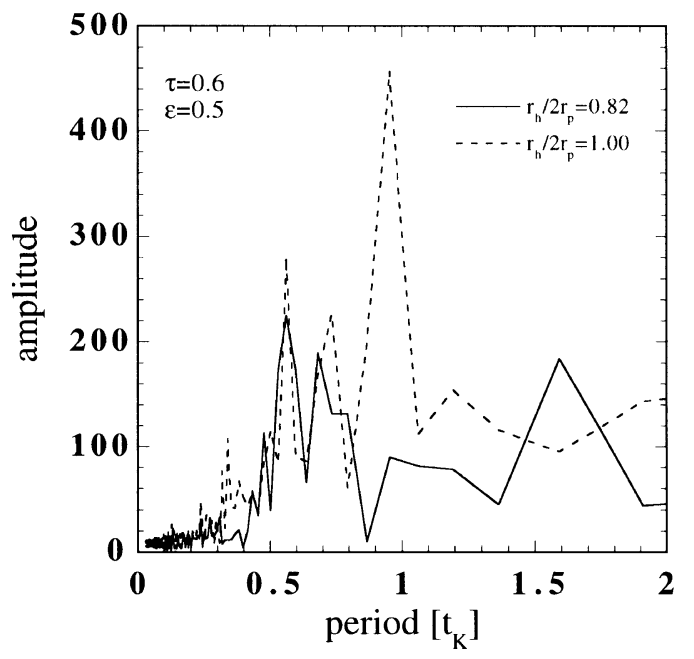

Fig. 7. Influence of simulation parameters on a period of oscillation observed in the evolution curve of $\left\langle v_{x}^{2}\right\rangle^{1 / 2}$. (a) Various numbers of particles but the same parameters of $\tau=1.2, \epsilon=0.4$, and $r_{\mathrm{h}} / 2 r_{\mathrm{p}}=0.675$. (b) Various optical depth but the same parameters of $\epsilon=0.4$, and $r_{\mathrm{h}} / 2 r_{\mathrm{p}}=0.675$. Simulations are done with the same simulation region as $L=59.3 r_{\mathrm{h}}(L=80 \mathrm{~m})$ and optical depth is controlled by changing number of particles. Used particles in each simulation are $N=1630$ for $\tau=0.8, N=2038$ for $\tau=1.0$, and $N=2444$ for $\tau=1.2$, respectively. (c) Various restitution coefficients but the same parameters of $\tau=1.1$ and $r_{\mathrm{h}} / 2 r_{\mathrm{p}}=0.675$. Simulation region is the same as the case of (b), but number of particles is $N=2240$. (d) Various $r_{\mathrm{h}} / 2 r_{\mathrm{p}}$ but the same parameters of $\tau=0.6$ and $\epsilon=0.5$. Width of simulation region and number of particles are $L=68.2 r_{\mathrm{h}}(L=112 \mathrm{~m})$ and $N=2400$ for $r_{\mathrm{h}} / 2 r_{\mathrm{p}}=0.82$ and $L=70.0 r_{\mathrm{h}}(L=140 \mathrm{~m})$ and $N=3744$ for $r_{\mathrm{h}} / 2 r_{\mathrm{p}}=1.00$, respectively.

examine the effects of the boundary conditions, we perform simulations with various sizes of simulation domain in the structured case, fixing $\tau=1.2, \epsilon=0.4$, and $r_{\mathrm{h}} / 2 r_{\mathrm{p}}=0.675$ (see Table 3 ). Figure 5 shows the equilibrium velocities as a function of number of particles $N$ (equivalently, the size of the simulation domain). Basic features are the same in all simulations, although it seems that $\left\langle v_{x}^{2}\right\rangle^{1 / 2}$ increases slightly with the increase of $N$ (simulation domain). The time evolution curves of $\left\langle v_{x}^{2}\right\rangle^{1 / 2}$ also show oscillation with a similar amplitude and a period.

Salo (1995) showed similar oscillation of $\left\langle v_{x}^{2}\right\rangle^{1 / 2}$ but the oscillation is not well resolved and recognized as "fluctuation". The reason is that the interval of his sampling time was large ( $\sim 0.5 t_{\mathrm{K}}$ in figure 2 in Salo (1995), in our simulations the interval is $\left.0.016 t_{\mathrm{K}}\right)$. We measure a period of the oscillation by Fourier transform methods. Figure 6 shows the results of this spectrum analysis in the non-structured and the structured cases. We find a strong peak at $\sim 0.6 t_{\mathrm{K}}$ for $\tau=1.2$ but not for $\tau=0.3$. Figure 7(a) is the results with various computational region for $\tau=1.2$ and show similar peak period. Figures 7(b), 7(c), and 7(d) are the results of simulations for various $\tau, \epsilon$, and $r_{\mathrm{h}} / 2 r_{\mathrm{p}}$, with the other parameters fixed. The dependence of the period on $\tau$ and $\epsilon$ is small. But the change of ratio $r_{\mathrm{h}} / 2 r_{\mathrm{p}}$ affects the period of oscillation. Figure $7(\mathrm{~d})$ shows that the width of peak and the period become larger with the increase in $r_{\mathrm{h}} / 2 r_{\mathrm{p}}$. Simulations in the structured cases always exhibit such an oscillatory feature and it would be related with the property of motion of particles.

We analyze the concurrent evolution of the spatial and the velocity distributions in detail. Figures 8(a) and 8(b) show the time sequence of the spatial distribution of particles from $t=8.91 t_{\mathrm{K}}$ to $10.19 t_{\mathrm{K}}$ and the corresponding velocity 

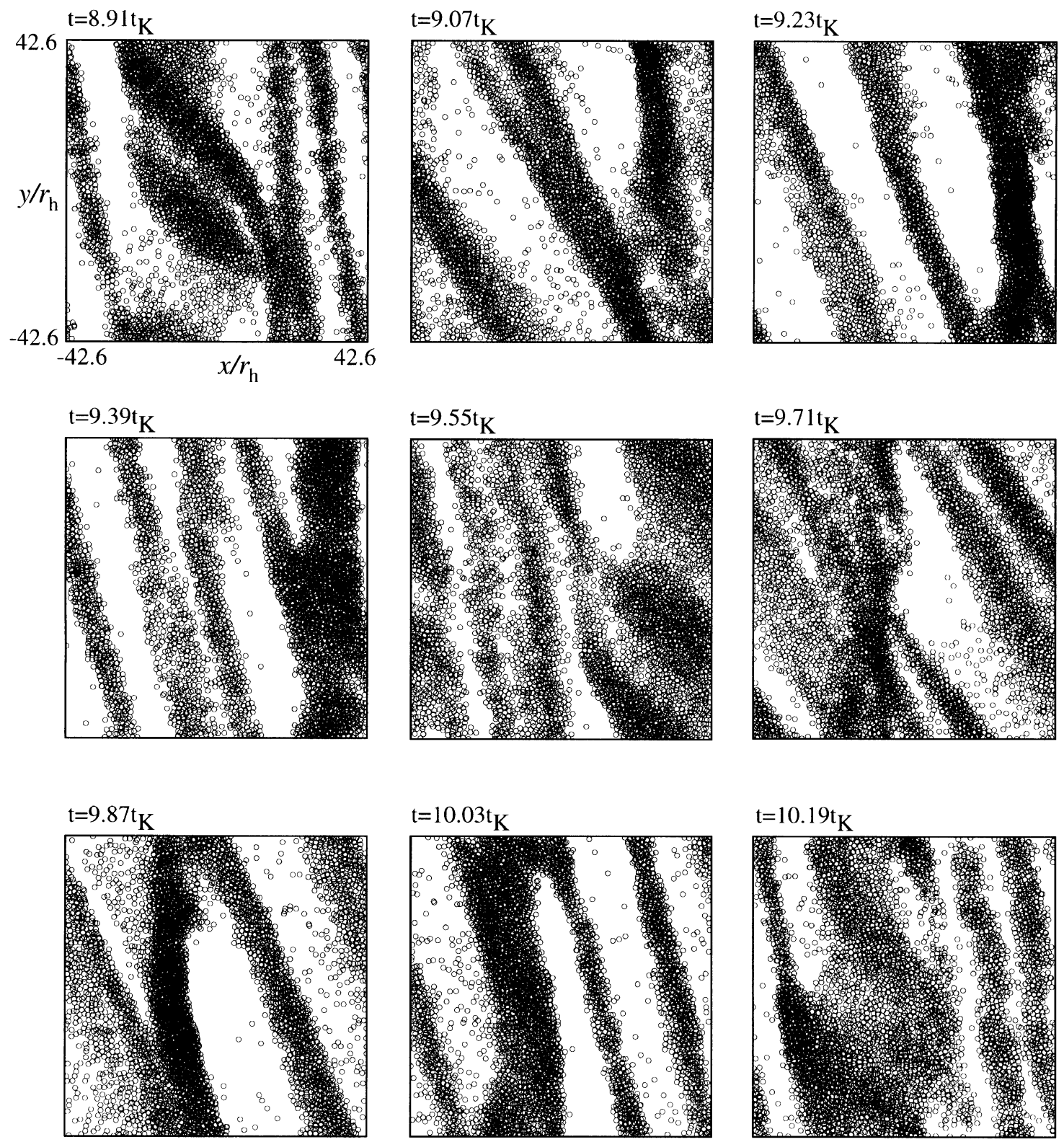

Fig. 8(a). Time sequence of spatial distribution of the structured case $(\tau=1.2)$ from $t=8.91 t_{\mathrm{K}}$ to $t=10.12 t_{\mathrm{K}}$. The width of region and number of particles are $L=115 \mathrm{~m}\left(L=85.2 r_{\mathrm{h}}\right)$ and $N=5052$, respectively.

distribution in the case of $\tau=1.2$.

As seen in Fig. 8(a), temporal clumps are formed due to gravitational instability and dissolved due to shearing motion. The change of spatial structure looks like turbulent fluid, where vortices are formed and dissolved successively. Velocity distribution as well as spatial distribution is not steady. Figure 8(b) shows that the velocity distribution revolves clockwisely, holding bar-like configuration. The configuration is similar at $t=8.91,9.55$, and $10.19 t_{\mathrm{K}}$, so that the period of the revolution is about $1.3 t_{\mathrm{K}}$. The oscillation in the evolution curve of $\left\langle v_{x}^{2}\right\rangle^{1 / 2}$ in the structured case observed in Fig. 4 would come from revolution of $v_{x}$-distribution. The periods obtained by the Fourier analysis of the evolution curve of $\left\langle v_{x}^{2}\right\rangle^{1 / 2}$ (Fig. 7) and in the velocity distribution are different by just a factor 2 . This is why $\left\langle v_{x}^{2}\right\rangle^{1 / 2}$ is mean square of $v_{x}$, i.e., the velocity dispersion takes maximum value twice during a period of the revolution of the configuration in velocity space. On the other hand, such change in the velocity distribution is not observed in the non-structured case. Figure 9 shows the velocity distribution for $\tau=0.3$ at $t=14.3 t_{\mathrm{K}}$. The distribution is independent of time.

In the theoretical studies (e.g., Goldreich and Tremaine, 1978; Araki and Tremaine, 1986), distribution function was assumed to be Gaussian distribution. In the optically thin case ( $\tau \ll 1), N$-body simulation showed that velocity distribution is Gaussian (Ida and Makino, 1992). The cumulative distributions of $v_{x}$ at different three time of $t=9.55,9.71$, and $9.87 t_{\mathrm{K}}$ in the structured case with $\tau=1.2$ are shown in Fig. 8(c) and that at $t=11.1,12.7$, and $14.3 t_{\mathrm{K}}$ in the non-structured case with $\tau=0.3$ are shown in Fig. 9. The velocity distribution is always given by Gaussian distribution in the latter case while it deviates from Gaussian distribution 

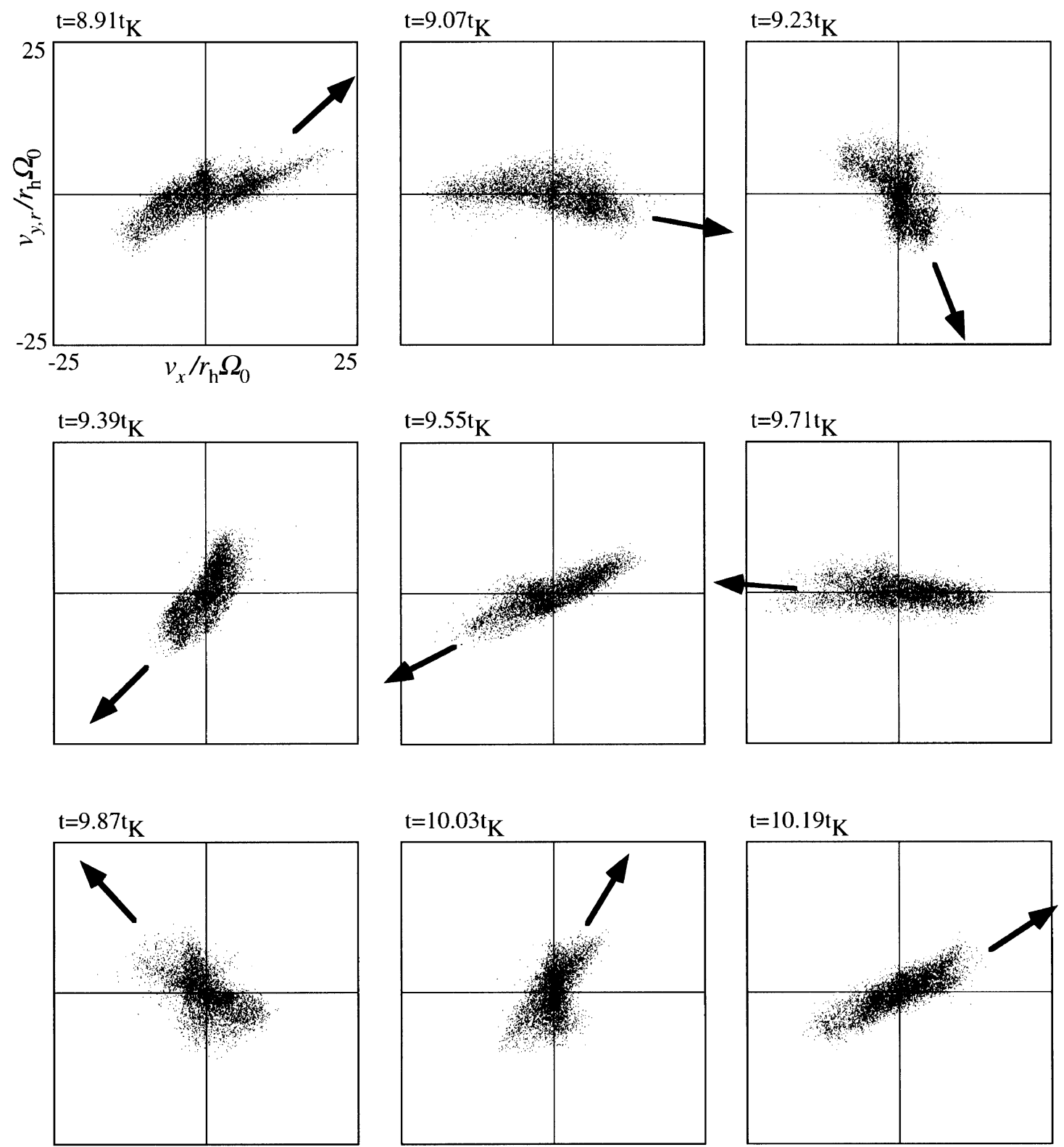

Fig. 8(b). Time sequence of the corresponding velocity distribution to Fig. 8 (a). For each particle, the local shear velocity $\left(0,3 \Omega_{0} x_{i} / 2,0\right)$ is subtracted. Arrows represent the direction of major long axis of distribution at each time.

in the former case. With the Kolmogonov-Smirnov test (see, e.g., Press et al., 1986), we calculate the probability which represents the similarity between a distribution and Gaussian distribution. The probability is $P=0.64$ at $t=11.1 t_{\mathrm{K}}$, $P=0.38$ at $t=12.7 t_{\mathrm{K}}$, and $P=0.41$ at $t=14.3 t_{\mathrm{K}}$, respectively, in the latter case. If $P=1$, two distributions are the same but if $P \ll 1$ they are different. In the structured case, $P=8.18 \times 10^{-13}$ at $t=9.55 t_{\mathrm{K}}, P=1.52 \times 10^{-20}$ at $t=9.71 t_{\mathrm{K}}$, and $P=5.52 \times 10^{-70}$ at $t=9.87 t_{\mathrm{K}}$, respectively. Thus our results show that in the non-structured case the velocity distribution of $v_{x}$ is expressed by Gaussian distribution with high accuracy but in the structured case the distribution is far from Gaussian.

How do individual particles behave in the wake-like structure? The $x$-component of position and the velocity of a Keplerian particle $i$ relative to the local shear velocity are written as (Nakazawa and Ida, 1988)

$$
\begin{aligned}
& x_{i}=a_{0} b_{i}-a_{0} e_{i} \cos \left(\Omega_{0} t-\delta_{i}\right), \\
& v_{x, i}=a_{0} \Omega_{0} e_{i} \sin \left(\Omega_{0} t-\delta_{i}\right), \\
& v_{y, r, i}=\frac{1}{2} a_{0} \Omega_{0} e_{i} \cos \left(\Omega_{0} t-\delta_{i}\right),
\end{aligned}
$$

where $e_{i}$ and $\delta_{i}$ are the eccentricity and the longitude of perihelion, and $a_{0}\left(1+b_{i}\right)$ is the semimajor axis of particle $i$. $v_{y, r, i}$ denotes $y$-component of velocity subtracted the local shear velocity, $v_{y, r, i}=v_{y, i}+3 \Omega_{0} x_{i} / 2$. If motion of particles is true Keplerian, orbital elements such as $e_{i}, \delta_{i}$ and $b_{i}$ are constants and the evolution curve of $x\left(v_{x}\right)$ of each particle represents a regular oscillation in $x\left(v_{x}\right)$ with a period of $1 t_{\mathrm{K}}$ and with an amplitude of $e a_{0}\left(e \Omega_{0} a_{0}\right)$. Mutual interactions such as direct collisions and self-gravity should cause motion 


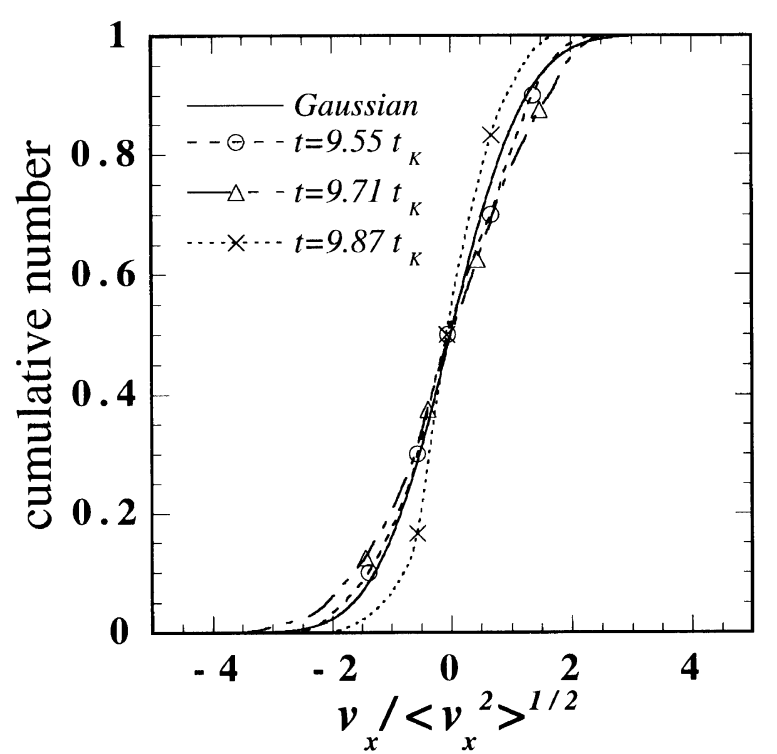

Fig. 8(c). Cumulative distribution of the radial velocity $v_{x}$ in Fig. 8(b). Broken line with open circle, dashed-dotted line with open triangle, and dotted line with cross denote distributions at $t=9.55 t_{\mathrm{K}}, t=9.71 t_{\mathrm{K}}$, and $t=9.87 t_{\mathrm{K}}$, respectively. Solid line denotes cumulative Gaussian distribution $P(s)=(1+\operatorname{erf}(s / \sqrt{2})) / 2$, where $\operatorname{erf}(s)$ is the error function. The distributions are scaled by the velocity dispersions at each time. The instantaneous velocity dispersion is $\left\langle v_{x}^{2}\right\rangle_{\text {ins }}^{1 / 2} / r_{\mathrm{h}} \Omega_{0}=6.100$ at $t=9.55 t_{\mathrm{K}}$, $\left\langle v_{x}^{2}\right\rangle_{\text {ins }}^{1 / 2} / r_{\mathrm{h}} \Omega_{0}=7.115$ at $t=9.71 t_{\mathrm{K}}$, and $\left\langle v_{x}^{2}\right\rangle_{\text {ins }}^{1 / 2} / r_{\mathrm{h}} \Omega_{0}=4.033$ at $t=9.87 t_{\mathrm{K}}$, respectively. The time averaged velocity dispersion is $\left\langle v_{x}^{2}\right\rangle_{\text {ave }}^{1 / 2} / r_{\mathrm{h}} \Omega_{0}=5.862$. The cumulative number is scaled by the total number of particles.

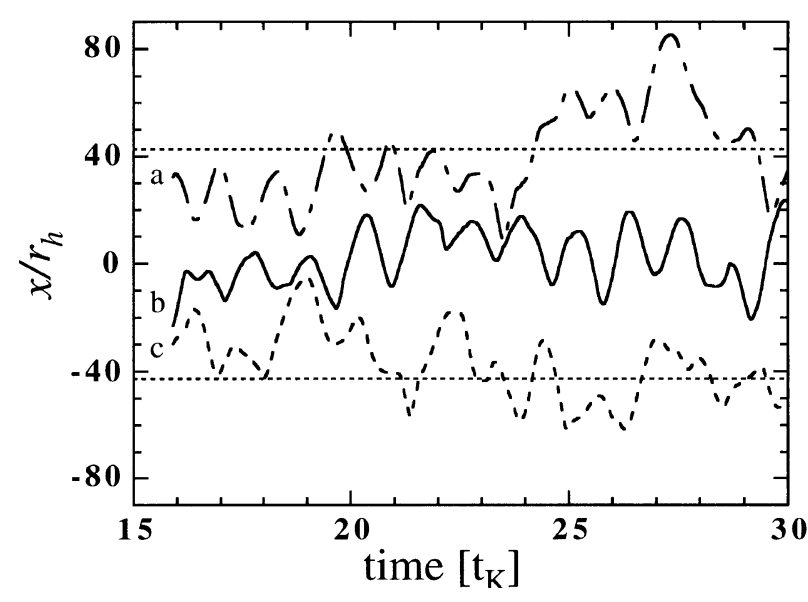

Fig. 8(d). Time evolutions of $x$-component of position for certain three particles in the structured case $(\tau=1.2)$. Solid, broken, and line-dotted lines illustrate the evolution curves of particles. Dotted lines denote positions of boundary of $x$-direction, the range of simulation domain is $\left[-42.62 r_{\mathrm{h}}, 42.62 r_{\mathrm{h}}\right]$. In this figure, curves are connected continuously by taking into account boundary conditions. But in fact, evolution curve for a particle over a boundary discontinues there and the curve corresponding to incoming particle starts from the opposite side.

of particle to deviate from true Keplerian orbit. Figures 8(d) and $8(\mathrm{e})$ show the time evolutions of $x$-components of position and velocity of arbitrary chosen three particles from $t=16 t_{\mathrm{K}}$ to $t=30 t_{\mathrm{K}}$ in the structured case used in Fig. 8(a). On timescale of a few $t_{\mathrm{K}}$, regular oscillation which comes

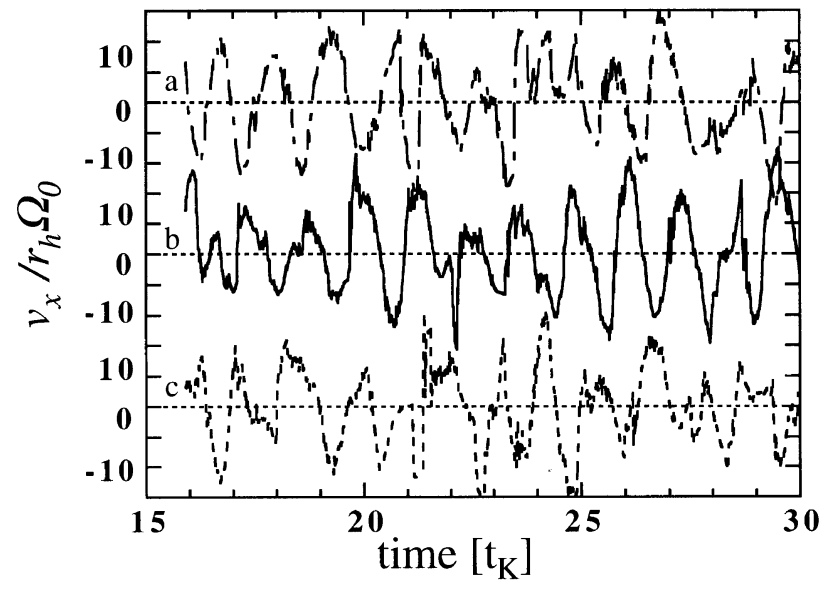

Fig. 8(e). Time evolutions of $v_{x}$ for particles used in Fig. 8(d). Each origin moves with appropriate shift and each dotted line denotes the line of $v_{x}=0$.

from Keplerian epicyclic motion is seen in both $x$ and $v_{x}$ but the period of this oscillation is slightly larger than $1 t_{\mathrm{K}}$. On longer timescale, the motion deviates from purely Keplerian orbit. We call such motion as "pseudo"-Keplerian motion.

The revolution of the velocity distribution with a period of an order of a Keplerian period observed in Fig. 8(b) would reflect "pseudo"-Keplerian motion of particles in the wake. From Eqs. (11), if perturbation by other particles is negligible, trajectory of a certain particle in velocity space is a ellipse with 2:1 axis ratio elongated to the $v_{x}$-direction expressed as $\left(v_{x, i} / a_{0} \Omega_{0} e_{i}\right)^{2}+\left(v_{y, r, i} /\left(\frac{1}{2} a_{0} \Omega_{0} e_{i}\right)\right)^{2}=1$ and the particle revolves in the clockwise direction. If all particles have similar phases $\delta_{i}$ or $\delta_{i}+\pi$, phase space motion would look like that in Fig. 8(b). This means that particles move coherently in the strong wake structure, holding the property of "pseudo"Keplerian motion. Self-gravity of particles may force their coherent motion with the mechanism proposed to maintain the elliptic ring around Uranus proposed by Goldreich and Tremaine (1979). If the motion is true Keplerian, the oscillation period should be equal to $1 t_{\mathrm{K}}$. But in the above structured case, the period observed in our simulations is about $1.3 t_{\mathrm{K}}$, apparently longer than $1 t_{\mathrm{K}}$. The increase in the period may be due to friction caused by inelastic collisions.

Next we analyze spatial distribution of particles, comparing it with the velocity distribution. In Fig. 10, we mark particles in 3 local spatial regions by filled circles, triangles, and squares and also mark the corresponding particles in the velocity space, both in non-structured and structured cases. Spatial distributions used here are the results in Fig. 4. In the non-structured case with $\tau=0.3$, the marked particles are scattered randomly in the velocity space, which means that there is no correlation of the velocity among neighbor particles, i.e., particles move almost randomly. But for $\tau=1.2$, as seen in Fig. 10(b), the neighbor particles in the real space are also localized in the velocity space. This indicates that neighbor particles have a similar velocity. Thus, in the wake, particles no longer move randomly but coherently (i.e., neighbors move together in the same direction at a similar velocity).

The coherent motion of particles is gradually destroyed 


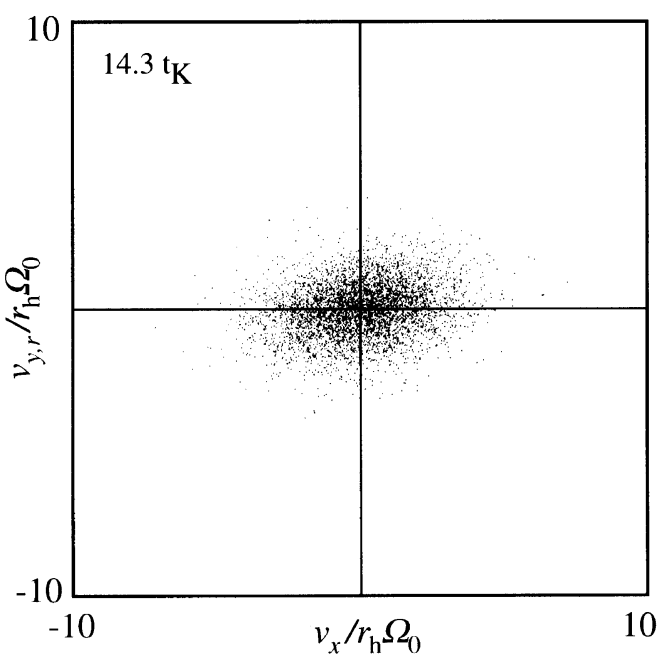

(a)

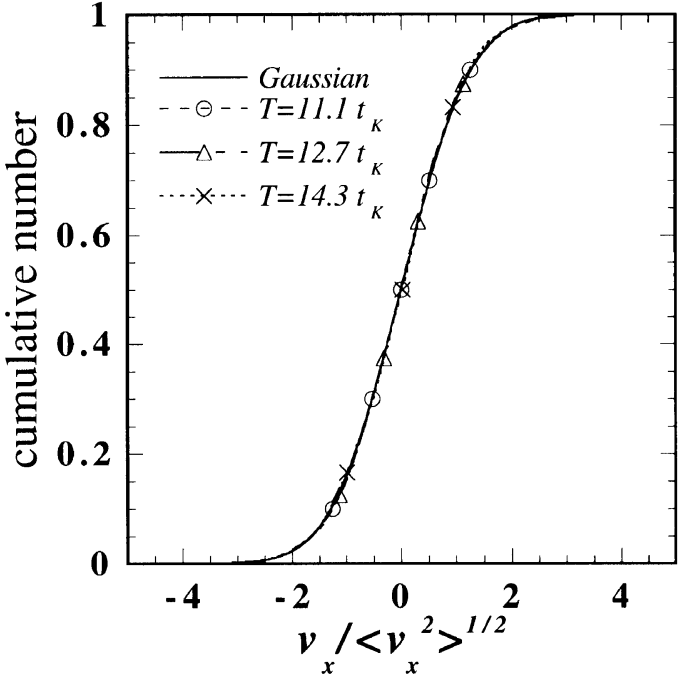

(b)

Fig. 9. Velocity distributions of the non-structured case $(\tau=0.3)$. (a) Particle distribution in velocity space at $t=14.3 t_{\mathrm{K}}$. (b) Cumulative distribution of radial velocity scaled by $\left\langle v_{x}^{2}\right\rangle^{1 / 2}$. The velocity dispersion is $\left\langle v_{x}^{2}\right\rangle^{1 / 2} / r_{\mathrm{h}} \Omega_{0}=1.720$. Broken line with open circle, dashed-dotted line with open triangle, and dotted line with cross denote distributions at $t=11.1 t_{\mathrm{K}}, t=12.7 t_{\mathrm{K}}$, and $t=14.3 t_{\mathrm{K}}$, respectively. Solid line denotes cumulative Gaussian distribution.

(a) non-structured case $\left(\tau=0.3, \mathrm{~L}=230 \mathrm{~m}, \mathrm{~L} / \mathrm{r}_{\mathrm{h}}=170.4\right)$
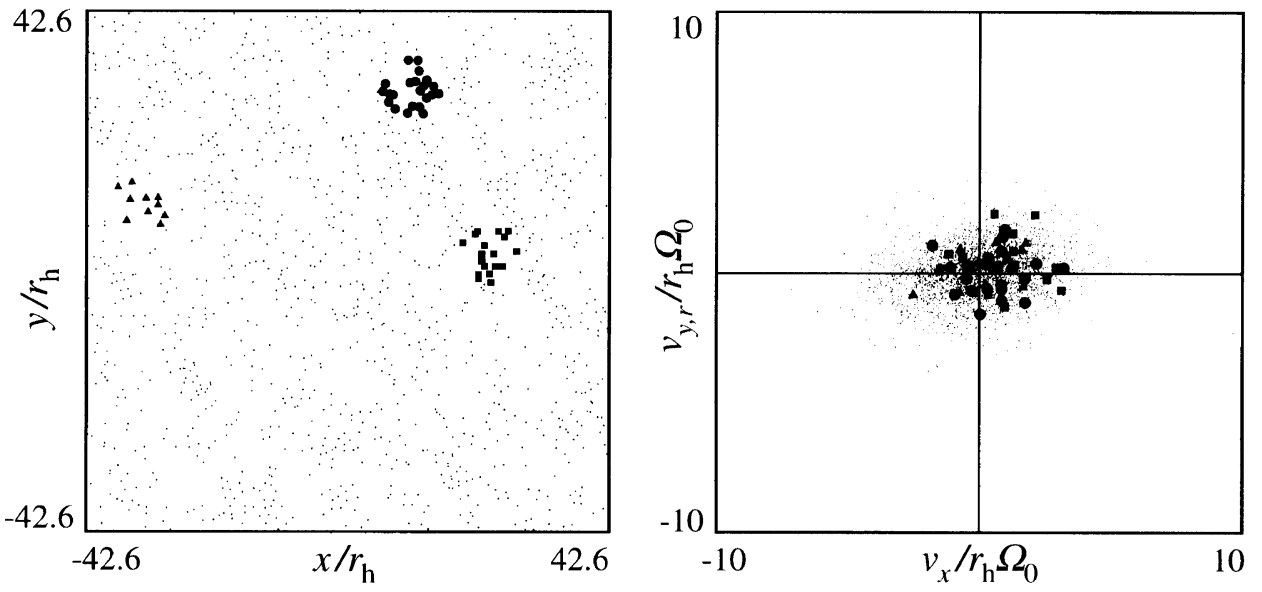

(b) structured case $\left(\tau=1.2, \mathrm{~L}=115 \mathrm{~m}, \mathrm{~L} / \mathrm{r}_{\mathrm{h}}=85.2\right)$
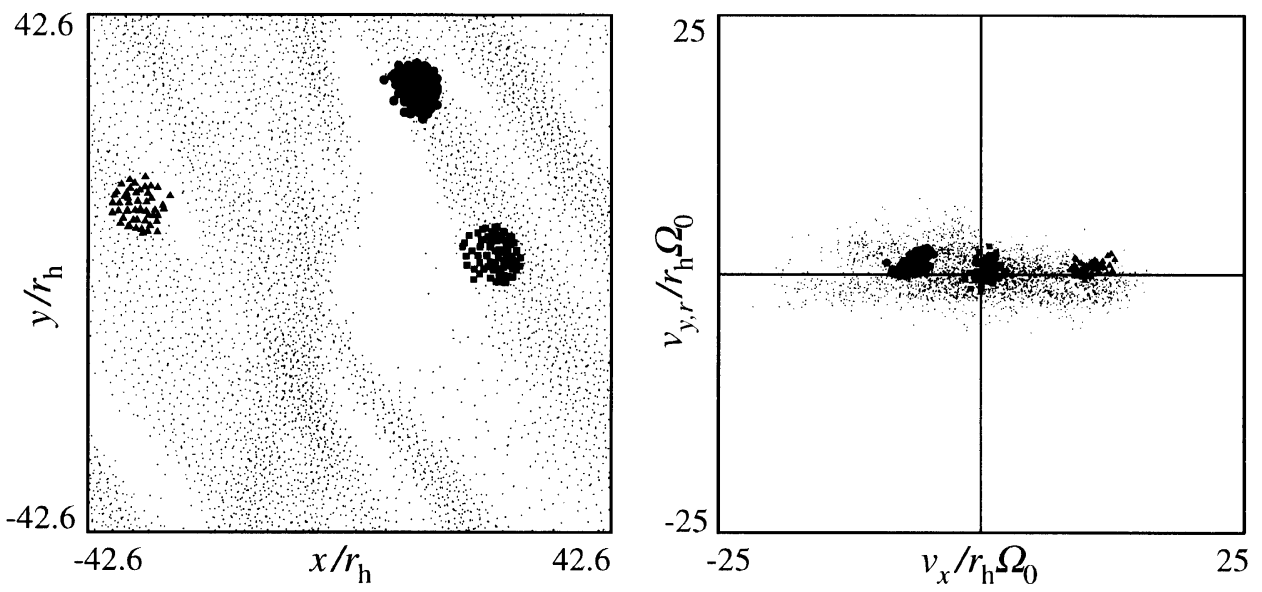

Fig. 10. The relation of marked particles in the real and the velocity space in the non-structured case $(\tau=0.3)(\mathrm{a})$ and structured case $(\tau=1.2)(\mathrm{b})$. Particles within certain three regions with the radius $5 r_{\mathrm{h}}$ which are located at $\left(-34 r_{\mathrm{h}}, 11 r_{\mathrm{h}}\right),\left(10 r_{\mathrm{h}}, 30 r_{\mathrm{h}}\right)$, and $\left(23 r_{\mathrm{h}}, 3 r_{\mathrm{h}}\right)$ in the real space are marked by symbols as filled triangle, circle, and square, respectively. The other particles are represented by small dots for convenience. In the non-structured case, edge of the spatial distribution is also trimmed. 
(a) non-structured case $(\tau=0.3)$

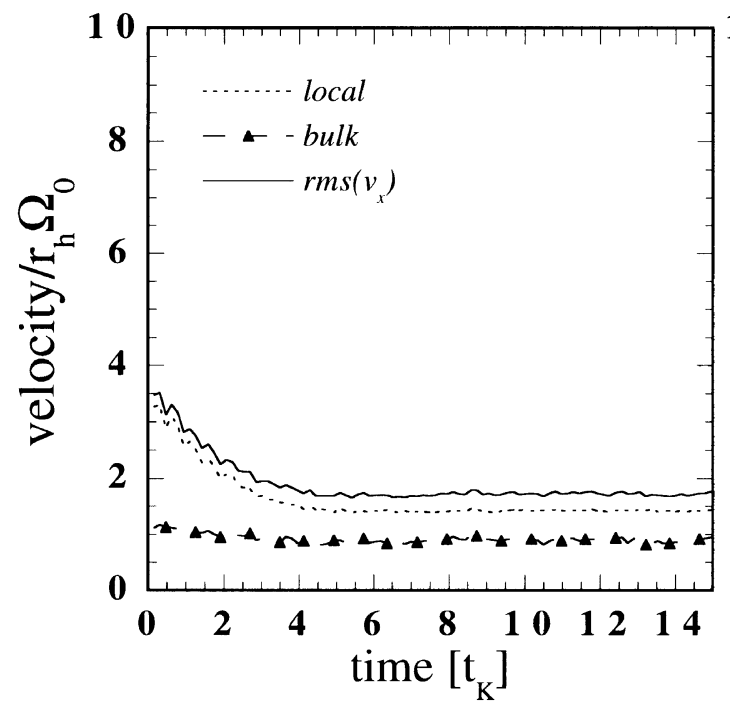

(b) structured case $(\tau=1.2)$

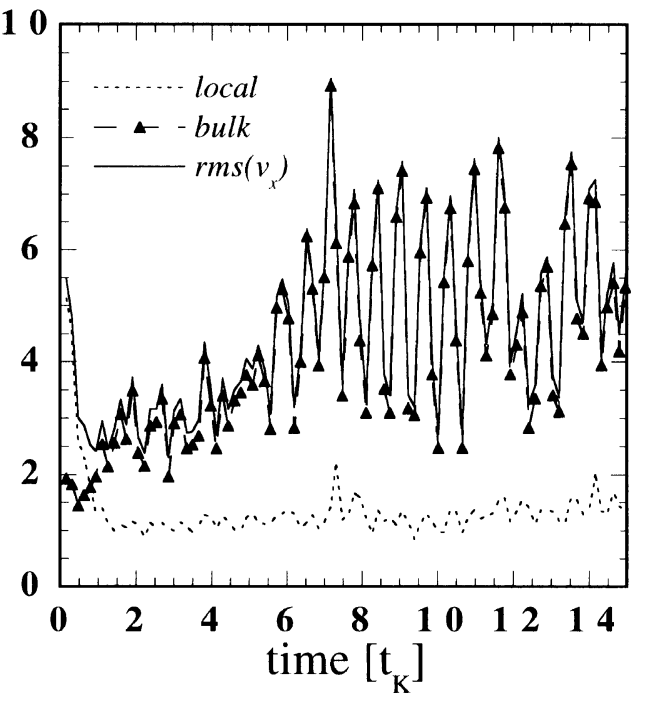

Fig. 11. Evolution curves of the bulk and local velocity dispersion in the non-structured case (a) and the structured case (b). Dotted line, dashed line with filled triangle, and solid line denote local, bulk velocity dispersion, and $\left\langle v_{x}^{2}\right\rangle^{1 / 2}$, respectively.

as the neighbor particles are separated from each other by shearing motion. We found that the marked particles diffuse in both space and velocity space on the time scale of an order of a Keplerian period. But a new coherent group is created from diffused distribution by the gravitational instability. Continuous creation and disruption of grouping occur in the wake.

The coherent motion affects the mean velocity of particles. The bulk velocity due to the coherent motion increases the radial velocity dispersion. Consider the population of particles marked by triangles in Fig. 10. In the non-structured case, triangles are distributed randomly in the velocity space so that the coherent motion is weak and the width of the distribution represents the randomness of motion. On the other hand, in the structured case triangles are distributed around a non-zero velocity. This means that triangles coherently move and have large bulk velocity. The spread of this region represents a magnitude of the local random velocity. We attempt to quantitatively separate the motion of a particle into the bulk and the local random motions following Salo (1995). Salo (1995) mainly considered the local random motion but did not study the bulk motion. The bulk motion is also important for understanding the wake structure, because the motion of particles is largely governed by the bulk motion. We consider both velocities in detail. We define the bulk velocity and the local random velocity of particle $i$ as the mean velocity averaged from the 10 nearest particles around particle $i$ and the difference from this mean velocity (use of more particles may include particles that are not coherently moving while less particles may cause large statistical fluctuation). These velocities are written as

$$
\begin{aligned}
& \boldsymbol{v}_{\mathrm{b}, i}=\frac{1}{10} \sum_{\substack{j \\
10 \text { nearest }}} \boldsymbol{v}_{j}, \\
& \boldsymbol{v}_{\mathrm{l}, i}=\boldsymbol{v}_{i}-\boldsymbol{v}_{\mathrm{b}, i} .
\end{aligned}
$$

The (total) velocity dispersion is expressed by using the bulk and the local (random) velocity dispersions as

$$
\left\langle v_{\alpha}^{2}\right\rangle \simeq\left\langle v_{\mathrm{b}, \alpha}^{2}\right\rangle+\left\langle v_{\mathrm{l}, \alpha}^{2}\right\rangle
$$

where $\alpha$ denotes components of $x, y, z$-direction. If there is no correlation of the velocity between neighbor particles, i.e., particles move randomly, $v_{\mathrm{b}, i} \simeq 0$ so that $\left\langle v^{2}\right\rangle^{1 / 2} \simeq$ $\left\langle v_{1}^{2}\right\rangle^{1 / 2}$, while if there is strong correlation, $\left|\boldsymbol{v}_{\mathrm{b}, i}\right| \gtrsim\left|\boldsymbol{v}_{1, i}\right|$ so that $\left\langle v^{2}\right\rangle^{1 / 2} \simeq\left\langle v_{\mathrm{b}}^{2}\right\rangle^{1 / 2}$.

Figure 11 shows the time evolution of $x$-component of the bulk and the local (random) velocity dispersions in the non-structured and the structured cases in Fig. 4. The local velocity dispersion is similar in both cases, initially the velocity dispersion decreases due to the collision damping to attain an equilibrium value with a small magnitude of fluctuation. On the other hand, the bulk velocity dispersion shows a quite different feature. In the non-structured case, bulk velocity is smaller than local velocity. This result reconfirms that in the non-structured case, particles move randomly but not systematically. But in the structured case, the bulk velocity grows and a large amplitude oscillation starts. The amplitude is far greater than the local velocity dispersion. In the structured case, the bulk velocity dominates the radial velocity dispersion. It is clear that the increase and the oscillation in the radial velocity dispersion are caused by bulk motion.

The distributions in the local and the bulk velocity spaces are also studied by the same method as in Fig. 10, in which neighbor particles are marked by the same symbol. Figure 12 separates the results in Fig. 10(b) into the bulk and the local random velocity components. The distribution of the local random components shows randomness of motion as in the non-structured case in Fig. 10(a) and there is no correlation between neighbor particles in the local random velocity. The shape of this distribution does not change with time. The local random velocity dispersion represents randomness of motion. The distribution of bulk component in Fig. 12(b) shows the clockwise revolution more clearly than in Fig. 8(b). 
(a) local

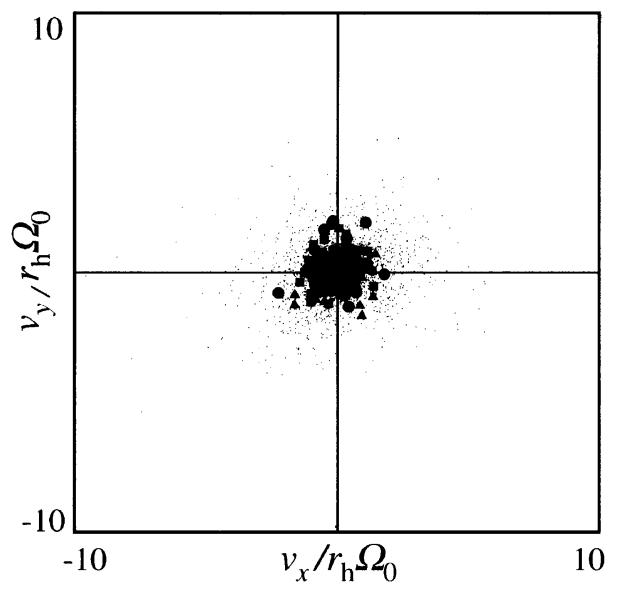

(b) bulk

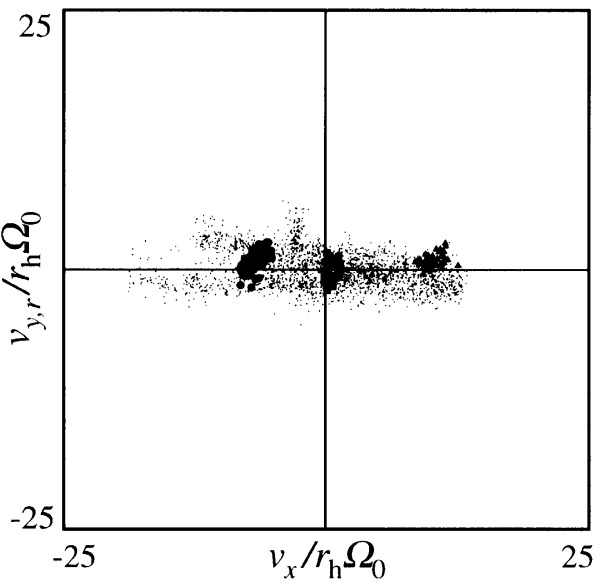

Fig. 12. The same as Fig. 10(b) but for particle distributions in the local and bulk velocity space. (a) Distribution for local velocity. (b) Distribution for bulk velocity. The local shear velocity of each particle is also eliminated.

\subsection{Characteristic scale of the wake structure in self- gravitating particles}

We also studied the characteristic scale of the wake structure. Number of particles used in Salo (1995) may not be enough to obtain the scale accurately. Although Griv (1998) performed local $N$-body simulations using $N=8000$ selfgravitating particles but without inelastic collision, he did not measure the scale. We analyze the characteristic scale of the wake through simulations with $N>5000$ up to 40000 particles.

Simulations were done for three different optical depth $\tau=0.3,0.6$, and 1.2 with fixed $\epsilon=0.4$ and $r_{\mathrm{h}} / 2 r_{\mathrm{p}}=$ 0.675 . For the first two simulations, the simulation region is a square and the width and number of particles are $L=230 \mathrm{~m}$ $\left(L=170.5 r_{\mathrm{h}}\right)$ and $N=5052$ for $\tau=0.3, L=150 \mathrm{~m}$ ( $L=118.6 r_{\mathrm{h}}$ ) and $N=4890$ for $\tau=0.6$. For $\tau=1.2$, we set the size of the simulation region and number of particles as $L_{x}=320 \mathrm{~m}\left(L_{x}=240 r_{\mathrm{h}}\right), L_{y}=350 \mathrm{~m}\left(L_{y}=260 r_{\mathrm{h}}\right)$, and $N=42780$. We adopted the autocorrelation analysis to determine the scale. The autocorrelation of the distribution function of particle position on $x-y$ plane $n(x, y)$ is defined by

$$
\operatorname{Corr}(r, s)=\int_{-\infty}^{\infty} d x \int_{-\infty}^{\infty} d y n(x+r, y+s) n(x, y)
$$

The distribution function $n(x, y)$ is given by Dirac's $\delta$ function $\delta(x)$ as

$$
n(x, y)=A \sum_{i} \delta\left(x-x_{i}\right) \delta\left(y-y_{i}\right)
$$

where $A$ is normalization factor and is chosen as $A=$ $\sqrt{L_{x} L_{y}} / N$ for later convenience. Substituting Eq. (15) into Eq. (14), we obtain

$$
\begin{aligned}
\operatorname{Corr}(r, s)= & \frac{L_{x} L_{y}}{N^{2}} \sum_{i} \sum_{j} \int_{-\infty}^{\infty} d x \delta\left(x+r-x_{j}\right) \delta\left(x-x_{i}\right) \\
& \times \int_{-\infty}^{\infty} d y \delta\left(y+s-y_{j}\right) \delta\left(y-y_{i}\right) .
\end{aligned}
$$

The integral in terms of $x$ is calculated as

$$
\int_{-\infty}^{\infty} d x \delta\left(x+r-x_{j}\right) \delta\left(x-x_{i}\right)= \begin{cases}1 & r=x_{j}-x_{i} \\ 0 & \text { otherwise }\end{cases}
$$

and the same calculation is done for the integral of $y$. This means that the right hand of Eq. (16) except for the coefficient represents the number of pairs of particles which satisfy the conditions $r=x_{j}-x_{i}$ and $s=y_{j}-y_{i}$, simultaneously. We take an average of Eq. (16) in terms of $r$ and $s$ in the range $[r, r+\Delta r]$ and $[s, s+\Delta s]$ as

$$
\begin{aligned}
\overline{\operatorname{Corr}(r, s)} & =\frac{1}{\Delta r \Delta s} \int_{r}^{r+\Delta r} d r^{\prime} \int_{s}^{s+\Delta s} d s^{\prime} \operatorname{Corr}\left(r^{\prime}, s^{\prime}\right), \\
& =\frac{L_{x} L_{y}}{\Delta r \Delta s N^{2}} n_{p}(r, s)
\end{aligned}
$$

where $n_{p}(r, s)$ is number of pairs of particles which satisfy $r<x_{j}-x_{i}<r+\Delta r$ and $s<y_{j}-y_{i}<s+\Delta s$. $\Delta r$ and $\Delta s$ are given by using numbers of division of simulation region $N_{x}$ and $N_{y}$ as $\Delta r=L_{x} / N_{x}$ and $\Delta s=L_{y} / N_{x}$ and Eq. (18) is expressed as

$$
\overline{\operatorname{Corr}(r, s)}=\frac{n_{p}(r, s)}{N^{2} / N_{x} N_{y}} .
$$

This formula is the same as superposition of particle's distribution viewed from each particle. In the case where particles distribute homogeneously in $x-y$ plane, i.e., in the nonstructured case, $n_{p} \sim N^{2} / N_{x} N_{y}$ so that $\overline{\operatorname{Corr}(r, s)} \sim 1$.

Salo (1995) also adopted the correlation function of particle position by superposition of particle's distribution instead of Fourier transform. Using this analysis, Salo (1995) displayed the wake-like structure similar to gravitational wake in the non-colliding particle's system that was studied by Julian and Toomre (1966). He mainly studied the influence of the boundary conditions on the configuration of the wake-like structure. In the analysis of the autocorrelation function, we adopt the same method used by Salo (1995). Figure 13 shows the autocorrelation functions of particle position and the particle distribution used in this analysis. For $\tau=0.3$, inhomo- 
(a)

$$
\text { amplitude }
$$

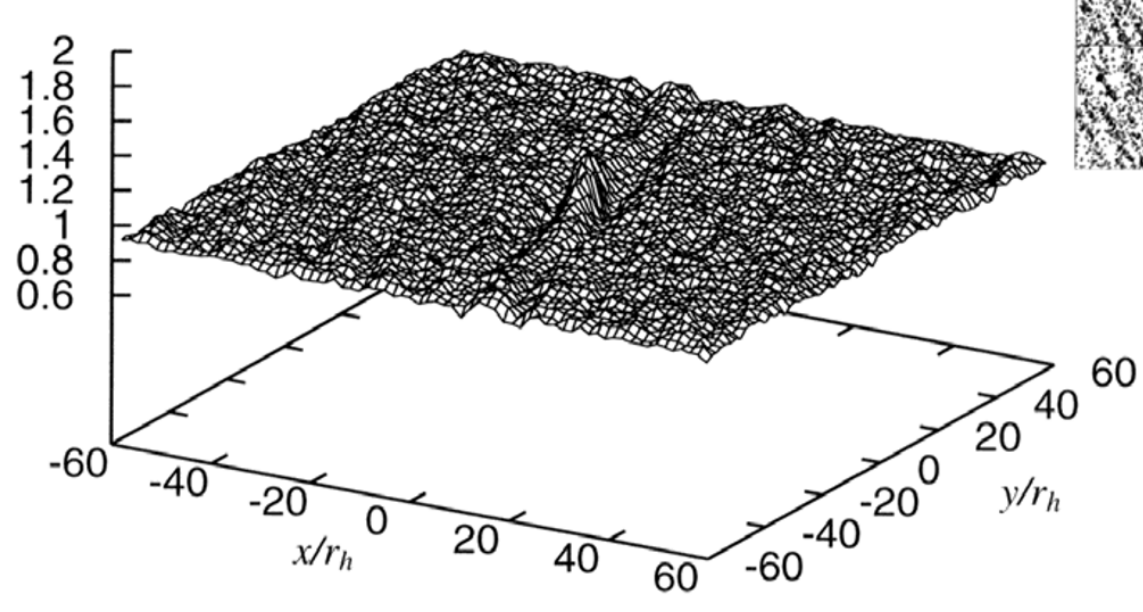

(b) amplitude
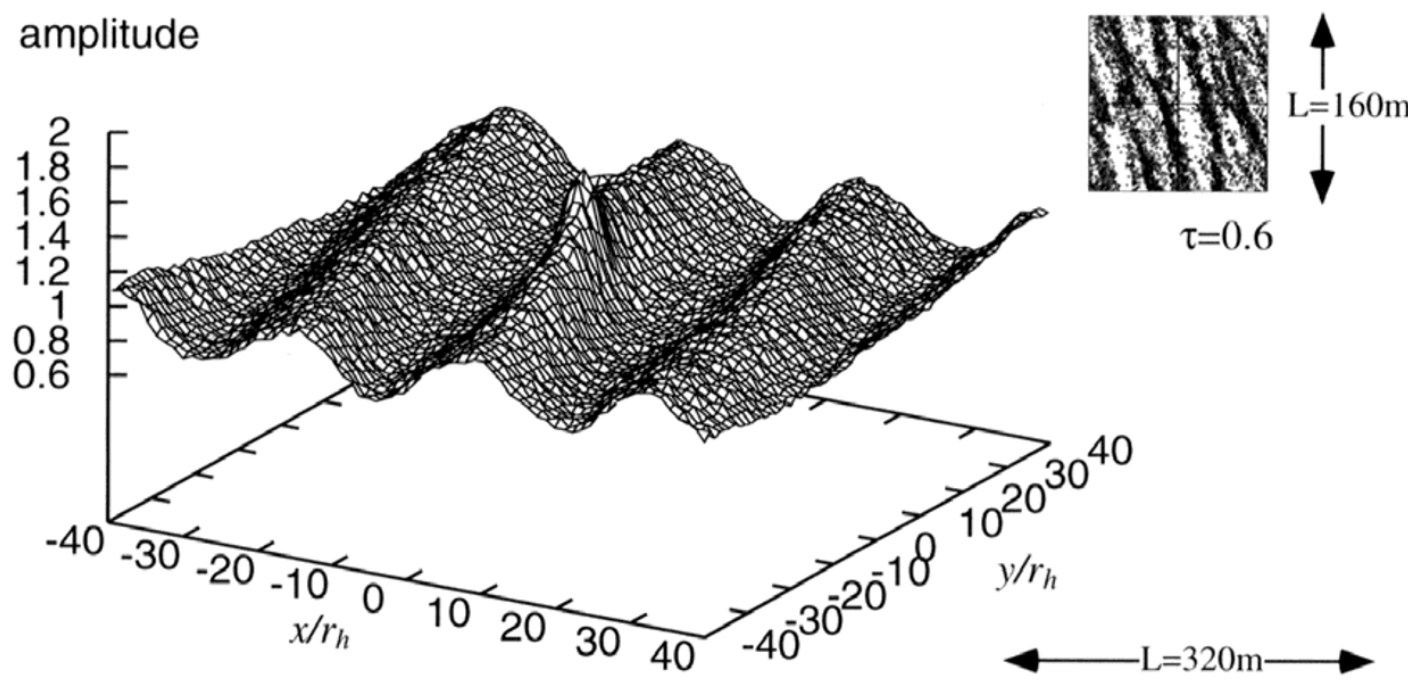

$\tau=0.6$

(c) amplitude

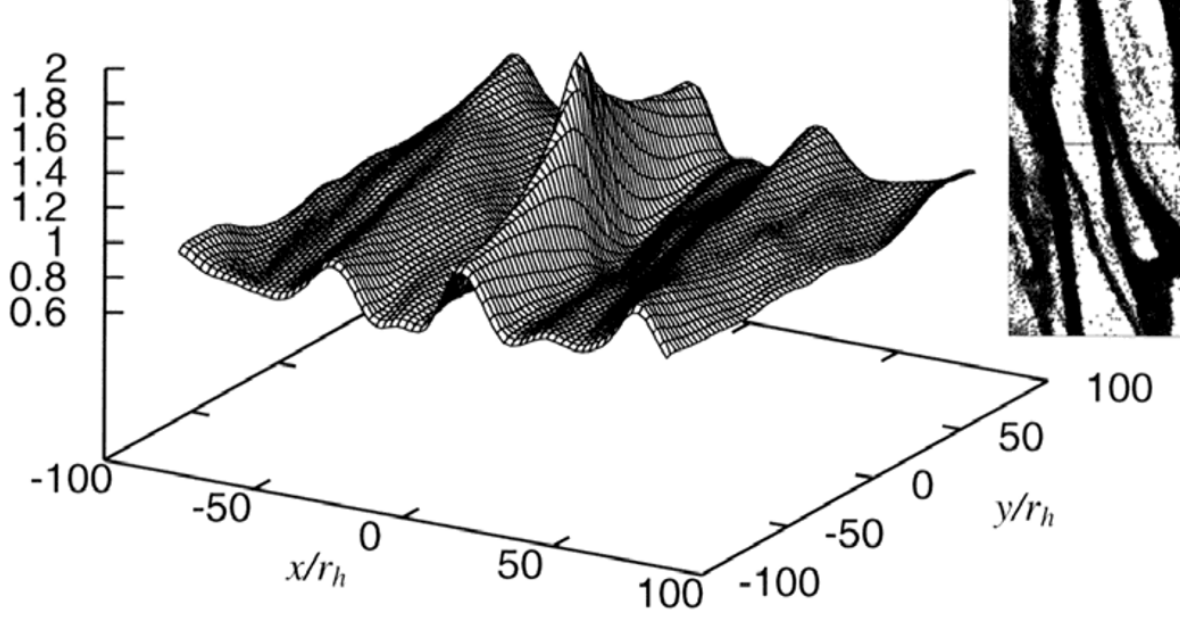

Fig. 13. Autocorrelation functions of particle positions in the case of $\tau=0.3$ (a), 0.6 (b), and 1.2 (c). In all simulations, $\epsilon=0.4$ and $r_{\mathrm{h}} / 2 r_{\mathrm{p}}=0.675$ are set up. We also showed the original snapshots used in this analysis.

geneity cannot be seen in particle distribution. The autocorrelation function does not have any clear structure without a peak near origin which is caused by temporary gravitational binding pointed out by Salo (1995). On the other hand, as $\tau$ increases, a wave-like structure gradually appears. For $\tau=0.6$ and 1.2 , several clear waves form in the autocorrelation functions. The shape of the waves slightly changes with time but the characteristic scale does not change. Figure 14 shows the cross sections at $y=$ constant for $\tau=0.6$ and 1.2. The cross sections at different $y$ overlap each other 
(a) $\tau=0.6$

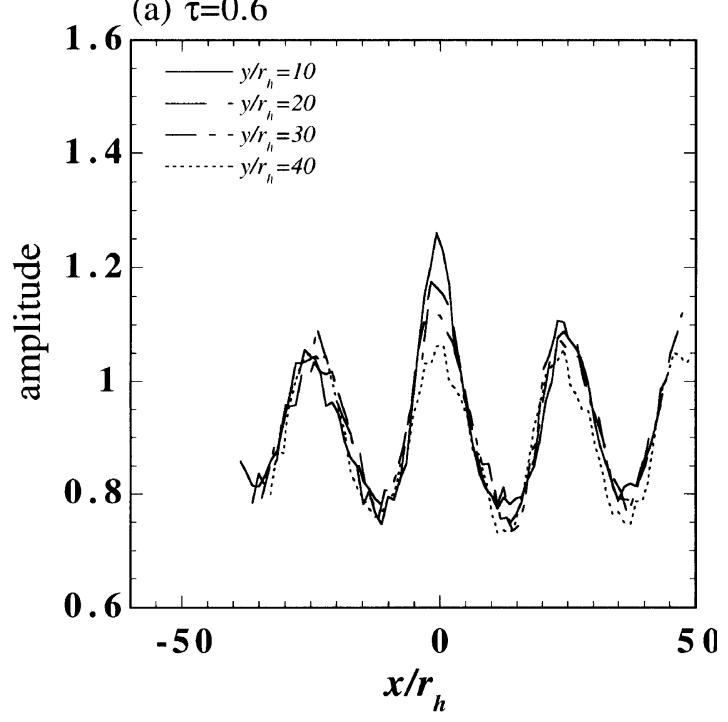

(b) $\tau=1.2$

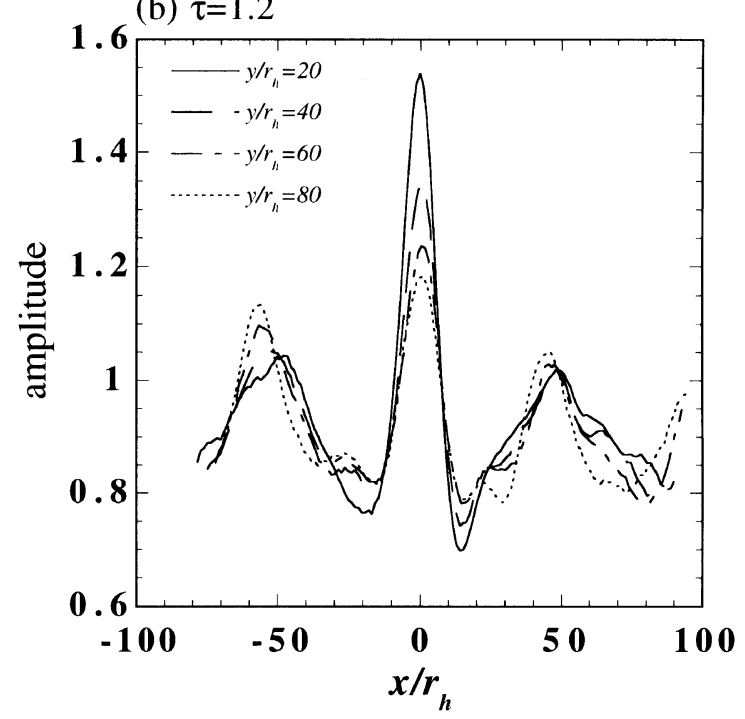

Fig. 14. Cross sections of autocorrelation functions for $\tau=0.6$ (a) and $\tau=1.2$ (b) in Fig. 13. Each cross section is shifted in the $x$-direction for peak positions to coincide with each other.

Table 4. Characteristic wavelength for large computational simulations.

\begin{tabular}{ccccccc}
\hline$\tau$ & $\lambda_{\text {ob }} / r_{\mathrm{h}}$ & $\lambda_{\text {cr }} / r_{\mathrm{h}}$ & $\lambda_{\text {un }}\left(\left\langle v_{x}^{2}\right\rangle^{1 / 2}\right) / r_{\mathrm{h}}$ & $\lambda_{\text {un }}\left(\left\langle v_{1, x}^{2}\right\rangle^{1 / 2}\right) / r_{\mathrm{h}}$ & $\left\langle v_{x}^{2}\right\rangle^{1 / 2} / r_{\mathrm{h}} \Omega_{0}$ & $\left\langle v_{1, x}^{2}\right\rangle^{1 / 2} / r_{\mathrm{h}} \Omega_{0}$ \\
\hline 0.3 & - & 10.31 & 22.67 & 15.56 & $1.72 \pm 0.0267$ & $1.42 \pm 0.014$ \\
0.6 & $\sim 25$ & 20.61 & 19.88 & 5.51 & $2.27 \pm 0.130$ & $1.20 \pm 0.037$ \\
1.2 & $\sim 50$ & 41.22 & 82.09 & 3.75 & $6.54 \pm 0.712$ & $1.40^{*}$ \\
\hline
\end{tabular}

${ }^{*}$ This value is not time-averaged.

with appropriate shifts. From these figures, we found that the characteristic wavelengths are $\lambda_{\mathrm{ob}} / r_{\mathrm{h}} \sim 25$ for $\tau=0.6$ and $\lambda_{\mathrm{ob}} / r_{\mathrm{h}} \sim 50$ for $\tau=1.2$, respectively.

Although these waves are the results from fully developed gravitational perturbation, we compare our results with the linear perturbation theory. We confirm that the scale of the wake structure is characterized by the longest wavelength $\lambda_{\text {cr }}$ that causes axisymmetric gravitational instability in a thin disk, as pointed out by Salo (1995). In the linear theory, the most unstable wavelength for a rotating and non-pressure sheet is (see, e.g., Binney and Tremaine, 1987)

$$
\lambda_{\text {un }}=\frac{v_{s}^{2}}{G \Sigma},
$$

where $v_{s}$ is the random velocity. When the wake structure is formed, $\left\langle v_{x}^{2}\right\rangle^{1 / 2}$ no longer represents random velocity because of the development of coherent motion. We use the local velocity dispersion $\left\langle v_{1, x}^{2}\right\rangle^{1 / 2}$ as well as $\left\langle v_{x}^{2}\right\rangle^{1 / 2}$ for the evaluation of Eq. (20). On the other hand, the wavelength $\lambda_{\text {cr }}$ is given as (Toomre, 1964)

$$
\lambda_{\mathrm{cr}}=\frac{4 \pi^{2} G \Sigma}{\kappa^{2}}
$$

where epicyclic frequency $\kappa$ is equal to the angular velocity $\Omega_{0}$ in Saturn's ring. By using parameters and values of the velocity dispersions obtained by our simulations, we evaluate the values of these wavelengths in both cases of $\tau=0.6$ and 1.2 and the results are listed in Table 4. The wavelengths $\lambda_{\text {un }}$ calculated from the local velocity dispersion are quite different from the results of simulations in all cases. For $\tau=1.2, \lambda_{\text {un }}$ calculated from $\left\langle v_{x}^{2}\right\rangle^{1 / 2}$ is two times larger than observed value and the dependence of $\tau$ seems to be different. On the other hand, $\lambda_{\text {cr }}$ agrees with the observed wavelength at all $\tau$. Thus, the scale of the wake structure is approximately determined by $\lambda_{\text {cr }}$ rather than $\lambda_{\text {un }}$. The result of this analysis reinforces Salo (1992b, 1995). For the B-ring, the wavelength calculated from Eq. (21) with Bring parameter $\Sigma=120 \mathrm{~g} / \mathrm{cm}^{3}$ (e.g., Esposito, 1993) is about $83 \mathrm{~m}$ for $\tau=1.0$ (we assumed $r_{\mathrm{p}}=1 \mathrm{~m}$ and $\rho=900 \mathrm{~kg} / \mathrm{m}^{3}$, respectively). This value is consistent with the observational scale $\sim 100 \mathrm{~m}$ obtained by PPS observation (Esposito et al., 1983a; Esposito, 1993).

\subsection{Velocity change along the line $\mathcal{Q} \simeq 2$}

Our simulations as well as those in Salo (1995) showed $\mathcal{Q} \simeq 2$ when the wake structure formed. The $\mathcal{Q}$-value is calculated by Eq. (1), using the equilibrium value of the radial velocity dispersion $\left\langle v_{x}^{2}\right\rangle^{1 / 2}$. But we showed that the motion of particles includes the large systematic motion and $\left\langle v_{x}^{2}\right\rangle^{1 / 2}$ no longer represents a magnitude of the random motion in the structured case. By using the local velocity dispersion, we obtain $\mathcal{Q}\left(\left\langle v_{1, x}^{2}\right\rangle^{1 / 2}\right) \simeq 0.38<2$ so that gravitational instability is very important (although ring particles do not 
form persistent clumps due to small $r_{\mathrm{h}} / 2 r_{\mathrm{p}}$ value).

In Fig. 11(b), the simulation starts with large random motion (large local velocity dispersion) to avoid gravitational instability. When the local velocity dispersion is reduced by the collisional dumping to the value for gravitational instability, $\mathcal{Q}\left(\left\langle v_{1, x}^{2}\right\rangle^{1 / 2}\right) \lesssim 2$, the bulk velocity dispersion starts to increase. The gravitational instability makes particles move coherently and the coherent motion dominates the radial velocity dispersion. The oscillation pattern comes from the coherent "pseudo"-Keplerian bulk motion. The mean value of the oscillating velocity becomes constant independent of time after $5 t_{\mathrm{K}}$. Using the mean value of the velocity dispersion, we obtain $\mathcal{Q} \simeq 2$. For simulations with different $\Sigma$ we also obtain $\mathcal{Q} \simeq 2$. That is, the mean velocity is larger for larger $\tau$.

However, we have not yet understood why $\mathcal{Q} \simeq 2$ is attained by the systematic motion. One possibility is gravitational scattering between the transient wakes, as Salo (1995) suggested. For the simplicity, we approximate the scattering efficiency by that between spherical clumps with mass $\lambda_{\mathrm{cr}}^{2} \Sigma$, which is typical mass of a clump formed by the selfgravitational instability. Gravitational perturbations pump up velocity dispersion to $\sim r_{h} \Omega_{0}$ at one encounter in the case of nearly circular non-inclined orbit (e.g., Ida, 1990). The $\mathcal{Q}$-value in this case is (see Eq. (1))

$$
\begin{aligned}
\mathcal{Q} \sim & \frac{\Omega_{0} \times r_{\mathrm{h}} \Omega_{0}}{3.36 G \Sigma}=\frac{\Omega_{0}^{2}}{3.36 G \Sigma}\left(\frac{\lambda_{\mathrm{cr}}^{2} \Sigma}{3 M_{s}}\right)^{1 / 3} \\
& \simeq 2.4
\end{aligned}
$$

Note that the dependences of $\mathcal{Q}$ on particle mass, $\Sigma, \Omega_{0}$, and $M_{s}$ are canceled by $\lambda_{\text {cr }}$ (see Eq. (21)) and $\mathcal{Q}$ takes a constant value $\sim 2$. When $\Sigma$ is larger, more massive clumps forms and velocity dispersion is increased by the stronger gravity. This result is consistent with the simulation results, however, more detail and more correct treatment is needed for the scattering between the wakes.

\section{Conclusion and Discussion}

In this study, we performed local $N$-body simulations including the mutual gravitational force between ring particles as well as the direct (inelastic) collision with identical (up to $N \simeq 40000$ ) particles. Salo (1995) showed that the spatial structures (wake and clumps) arise spontaneously in a dense, self-gravitating, and collisionally dumping particle system due to gravitational instability. He also showed that as the wake forms, the radial velocity dispersion increases with the relation $\mathcal{Q} \sim 2$, where $\mathcal{Q}$ is Toomre's non-dimensional parameter. We reconfirmed his results (Subsection 3.1). Furthermore, our simulations showed a regular oscillatory pattern in the velocity dispersion which was not found in Salo (1995). We analyzed the motion of particles in the wake structure in detail and clarified that the intrinsic physics of the regular oscillation is coherent "pseudo"-Keplerian motion induced by self-gravitational instability (Subsection 3.2).

The wake-like structure is not steady but changes with time, continuously forming clumps due to gravitational instability and dissolving due to shear motion, which looks like the turbulent fluid. We clearly showed that in such circumstances, particles no longer move randomly but coherently.
The coherent motion results in transient Keplerian motion with similar orbital elements of the particles in a clump, presumably because of mutual forcing by self-gravity. Such a forcing mechanism is proposed to explain the elliptic ring around Uranus (Goldreich and Tremaine, 1979). If we separate the particle motion into the bulk and the local random motions following Salo (1995), in the structured system, the motion is dominated by the bulk motion and the local random motion is small with almost steady distribution. Owing to the coherent motion, the radial velocity dispersion oscillates in a period of the order of the Keplerian period, but the period observed in our simulations is somewhat larger than one Keplerian period, which may be because of friction through inelastic collisions.

We obtained the wavelength of the wake through autocorrelation analysis in Subsection 3.3. The wavelength observed in our simulation is approximately given by the longest wavelength $\lambda_{\mathrm{cr}}$ in the linear theory of axisymmetric gravitational instability in a thin disk. The wavelength $\lambda_{\text {cr }}$ is given by $\lambda_{\text {cr }}=4 \pi^{2} G \Sigma / \kappa^{2}$ (Toomre, 1964). The gravitational scattering between the clumps with typical mass $\sim \Sigma \lambda_{\mathrm{cr}}^{2}$ may be responsible for $\mathcal{Q} \sim 2$ as shown in Subsection 3.4, however, we need more detailed analysis on non-linear effects of the strong wake structure.

The structure observed in our and Salo's simulations is non-axisymmetric one. The observation in Saturn's A-ring produced the evidence of the asymmetric structure which may be caused by the wake (Dones et al., 1993). The characteristic scale of the structure observed in our simulations corresponds to the size of an order of $\sim 100 \mathrm{~m}$ for B-ring's parameters, which is consistent with the scale of the microstructure observed by PPS observation. The microstructure may correspond to the wake structure we found. More information about structure of the ring will be obtained by Cassini mission.

Our simulation results could be applicable to other optically thick particulate disks in which both the mutual gravitational force between particles and the collisional dumping are important. One of such systems is the Uranian elliptic ring system. Uranian rings are also optically thick system (e.g., Esposito, 1993) and a wake-like structure may arise due to gravitational instability. In the framework of a giant impact model of the origin of moon, it is considered that an optically thick debris disk formed around proto-earth after the impact and that the moon would have accreted in the disk (Hartmann and Davis, 1975; Cameron and Ward, 1976). According to $N$-body simulations performed by Ida et al. (1997) and Kokubo et al. (1999), spiral patterns develop in the early stage of the simulation and it results in rapid transfer of angular momentum and mass.

In the next paper, we will address angular momentum transfer enhanced by gravitational torque of the wake structure in detail.

Acknowledgments. The authors are indebted to S. Araki and $\mathrm{K}$. Ohtsuki for their variable comments and useful suggestions. We thank H. Tanaka, H. Emori, and K. Nakazawa for continuous encouragement. We also thank J. Makino and E. Kokubo for technical advice about HARP-2. This work was supported by the Grant-inAid of the Japanese Ministry of Education, Science, Sports, and Culture (09440089). 


\section{References}

Araki, S., The dynamics of particles disks. II. Effects of spin degrees of freedom, Icarus, 65, 83-109, 1988.

Araki, S., The dynamics of particles disks. III. Dense and spinning particle disks, Icarus, 90, 139-171, 1991.

Araki, S. and S. Tremaine, The dynamics of dense particle disks, Icarus, 65, 83-109, 1986.

Binney, J. and S. Tremaine, Galactic Dynamics, 283 pp., Princeton Univ. Press, Princeton, NJ, 1987.

Bridges, F. G., A. Hatzes, and D. N. C. Lin, Structure, stability and evolution of Saturn's rings, Nature, 309, 333-335, 1984.

Cameron, A. G. W. and W. R. Ward, The origin of the Moon, Proc. Lunar Planet Sci. Conf., 7, 120-122, 1976.

Dilley, J. P., Energy loss in collisions of icy spheres: Loss mechanism and size-mass dependence, Icarus, 105, 225-234, 1993.

Dones, L., J. N. Cuzzi, and M. R. Showalter, Voyager photometry of Saturn's A ring, Icarus, 105, 184-215, 1993.

Esposito, L. W., Understanding planetary rings, Annu. Rev. Earth Planet. Sci., 21, 487-523, 1993.

Esposito, L. W., M. O'Callaghan, and R. A. West, The structure of Saturn's rings: Implications from the Voyager stellar occultation, Icarus, 56, 439452, 1983a.

Esposito, L. W., M. O'Callaghan, K. E. Simmons, C. W. Hord, R. A. West, A. L. Lane, R. B. Pomphery, D. L. Coffeen, and M. Sato, Voyager photopolarimeter stellar occultation of Saturn's rings, J. Geophys. Res., 88, 8643-8649, 1983b.

Goldreich, P. and S. Tremaine, The velocity dispersion in Saturn's rings, Icarus, 34, 227-239, 1978.

Goldreich, P. and S. Tremaine, Precession of the $\epsilon$ ring of Uranus, Astron. J., 84, 1638-1641, 1979.

Goldreich, P. and S. Tremaine, The dynamics of planetary rings, Annu. Rev. Astron. Astrophys., 20, 249-283, 1982.

Griv, E., Local stability criterion for the Saturnian ring system, Planet. Space Sci., 46, 615-628, 1998.

Hartmann, W. K. and D. R. Davis, Satellite-sized planetesimals and lunar origin, Icarus, 24, 504-515, 1975.

Hatzes, A. P., F. G. Bridges, and D. N. C. Lin, Collision properties of ice spheres at low impact velocities, Mon. Not. R. Astron. Soc., 231, 1091$1115,1988$.

Hill, G. W., Researches in the lunar theory, Amer. J. Math., 1, 5-26, 129-147, 245-260, 1878.

Ida, S, Stirring and dynamical friction rates of planetesimals in the solar gravitational field, Icarus, 88, 129-145, 1990.

Ida, S. and J. Makino, $N$-body simulation of gravitational interaction between planetesimals and a protoplanet I. Velocity distribution of planetesimals, Icarus, 96, 107-120, 1992.

Ida, S., R. M. Canup, and G. R. Stewart, Lunar accretion from an impactgenerated disk, Nature, 389, 353-357, 1997.

Julian, W. H. and A. Toomre, Non-axisymmetric responses of differentially rotating disks of starts, Astrophys. J., 146, 810-827, 1966.

Kokubo, E., S. Ida, and J. Makino, Evolution of a circumterrestrial disk and formation of a single moon, Icarus, 1999 (submitted).

Lin, D. N. C. and P. Bodenheimer, On the stability of Saturn's rings,
Astrophys. J. Lett., 248, L83-L86, 1981.

Lukkari, J., Collisional amplification of density fluctuations in Saturn's rings, Nature, 292, 433-435, 1981.

Makino, J. and S. J. Aarseth, On a Hermite integrator with Ahmad-Cohen scheme for gravitational many-body problems, Publ. Astron. Soc. Japan., 44, 141-151, 1992.

Makino, J., E. Kokubo, and M. Taiji, HARP: A special-purpose computer for $N$-body problem, Publ. Astron. Soc. Japan., 45, 349-360, 1993.

Nakazawa, K. and S. Ida, Hill's approximation in the three-body problem, Prog. Theor. Phys. Suppl., 96, 167-174, 1988.

Ohtsuki, K., Equilibrium velocities in planetary rings with low optical depth, Icarus, 95, 265-282, 1992.

Ohtsuki, K., Capture probability of colliding planetesimals: Dynamical constraints on accretion of planets, satellites, and ring particles, Icarus, 106, 228-246, 1993.

Ohtsuki, K., Evolution of particle velocity dispersion in a circumplanetary disk due to inelastic collisions and gravitational interactions, Icarus, 137, 152-177, 1999.

Press, W. H., B. P. Flannery, S. A. Teukolsky, and W. T. Vetterling, Numerical Recipes, 472 pp., Cambridge Univ. Press, London/NewYork, 1986.

Richardson, D. C., Tree code simulations of planetary rings, Mon. Not. R. Astron. Soc., 269, 493-511, 1994.

Salo, H., Numerical simulations of dense collisional systems, Icarus, $\mathbf{9 0}$, 254-270, 1991.

Salo, H., Numerical simulations of dense collisional systems. II. Extended distribution of particle sizes, Icarus, 96, 85-106, 1992a.

Salo, H., Gravitational wakes in Saturn's rings, Nature, 395, 619-621, $1992 b$.

Salo, H., Simulations of dense planetary rings. III. Self-gravitating identical particles, Icarus, 117, 287-312, 1995.

Smith, B. A., L. Soderblom, R. Batson, P. Bridges, J. Inge, H. Masursky, E. Shoemaker, R. Beebe, J. Boyce, G. Briggs, A. Bunker, S. A. Collins, C. J. Hansen, T. V. Johnson, J. L. Mitchell, R. J. Terrile, A. F. Cook, II, J. Cuzzi, J. B. Pollack, G. E. Danielson, A. P. Ingersoll, M. E. Davies, G. E. Hunt, D. Morrison, T. Owen, C. Sagan, J. Veverka, R. Strom, and V. E. Suomi, A new look at Saturn system: The Voyager 2 images, Science, 215, 504-537, 1982.

Sugimoto, D., Y. Chikada, J. Makino, T. Ito, T. Ebisuzaki, and M. Umemura, A special-purpose computer for gravitational many-body problem, Nature, 345, 33-35, 1990.

Supulver, K. D., F. G. Bridges, and D. N. C. Lin, The coefficient of restitution of ice particles in glancing collisions: Experimental results for unfrosted surfaces, Icarus, 113, 188-199, 1995.

Toomre, A., On the gravitational stability of a disk of stars, Astrophys. J., 139, 1217-1238, 1964.

Ward, W. R., On the radial structure of Saturn's ring, Geophys. Res. Lett., 8, 641-643, 1981.

Wisdom, J. and S. Tremaine, Local simulations of planetary rings, Astron. J., 95, 925-940, 1988.

H. Daisaka (e-mail: hdaisaka@geo.titech.ac.jp) and S. Ida (e-mail: ida@geo.titech.ac.jp) 